\title{
ATLAS Trigger: design and commissioning
}
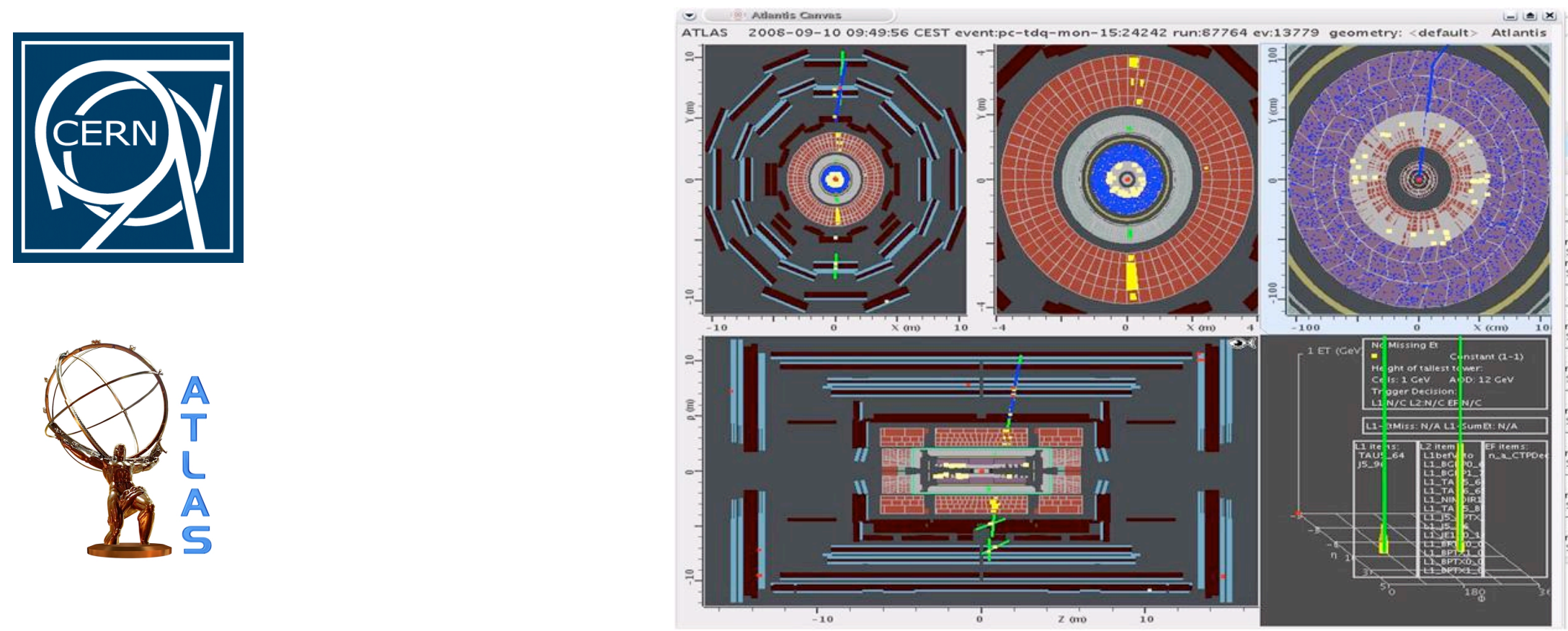

ICCMSE Symposium: Computing in Experimental High Energy Physics September 29th - October 4th 2009 Rhodes, Greece 


\section{Outline of the talk}

2) Motivations

Trigger architecture

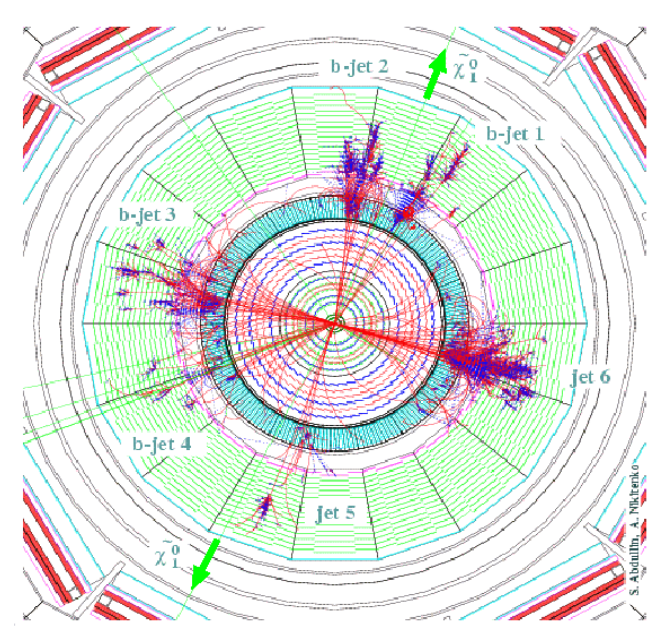

Trigger performance

7 Commissioning with cosmic rays and first beams

Trigger strategies and preparation for first collisions 


\section{The ATLAS detector @ LHC}

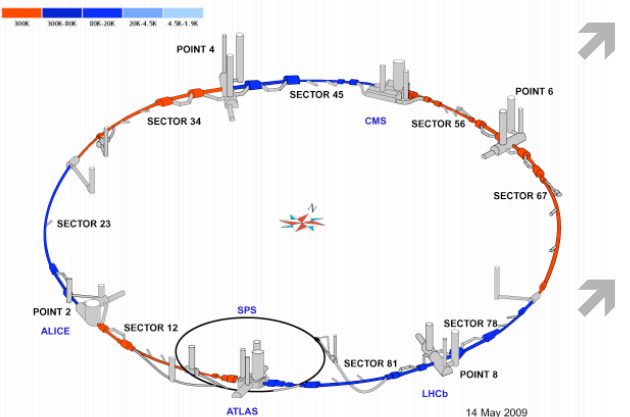

THC design parameters: $\mathrm{Vs}=14 \mathrm{TeV}$ and $\mathrm{L}=10^{34} \mathrm{~cm}^{-2} \mathrm{~s}^{-1}$

$\pi$ Providing $100 \mathrm{fb}^{-1} /$ year

$\pi$ Initially running at $7 \mathrm{TeV}$, rising towards $10 \mathrm{TeV}$, and much lower luminosity

Bunch-crossing rate $40 \mathrm{MHz}$ (25 ns bunch spacing)

$\pi$ Only $80 \%$ of bunched will be filled, effective bunch-crossing rate $32 \mathrm{MHz}$ $44 \mathrm{~m}$

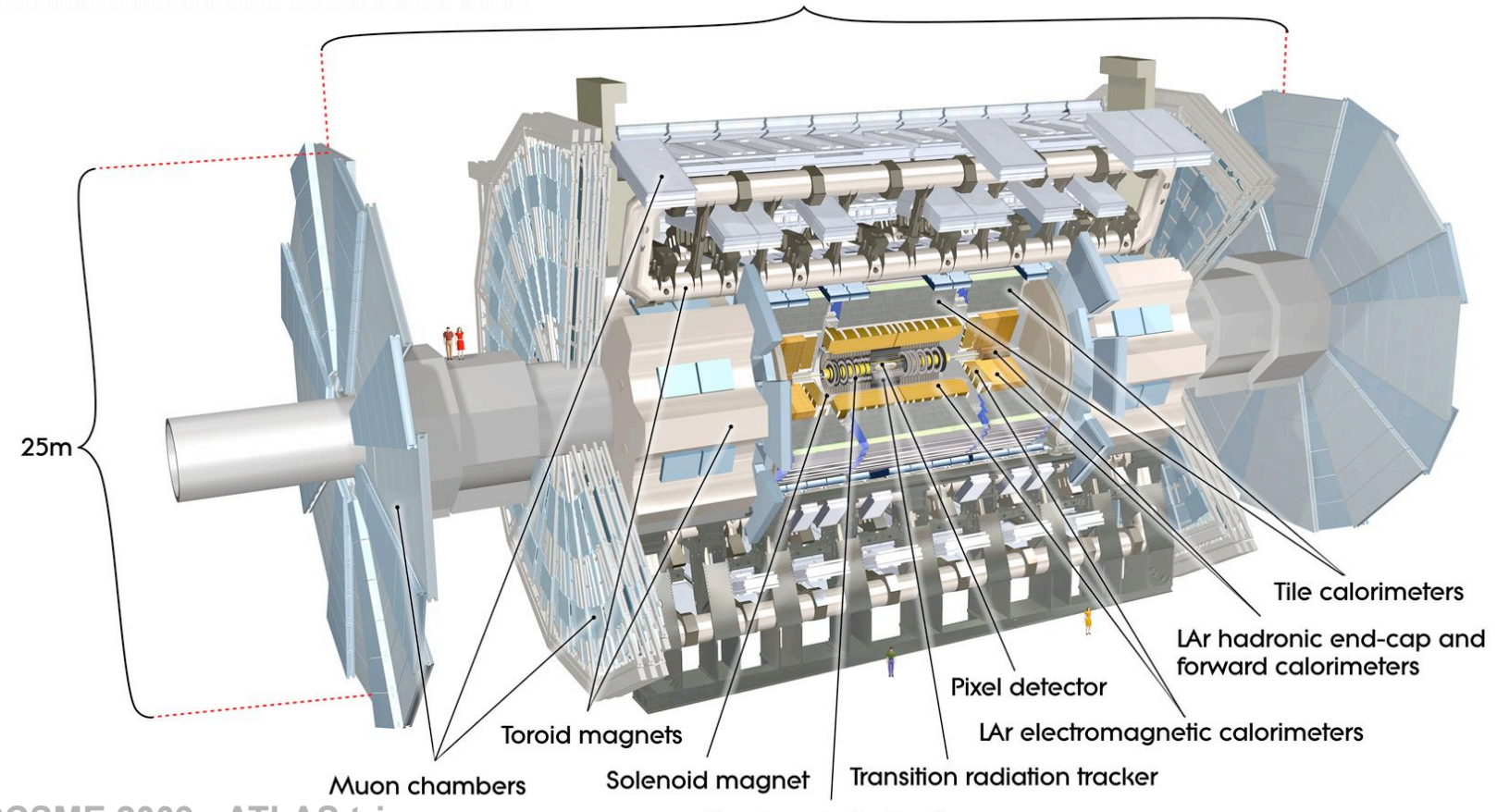

ATLAS is a generalpurpose detector to explore SM physics and beyond 


\section{Trigger motivations}

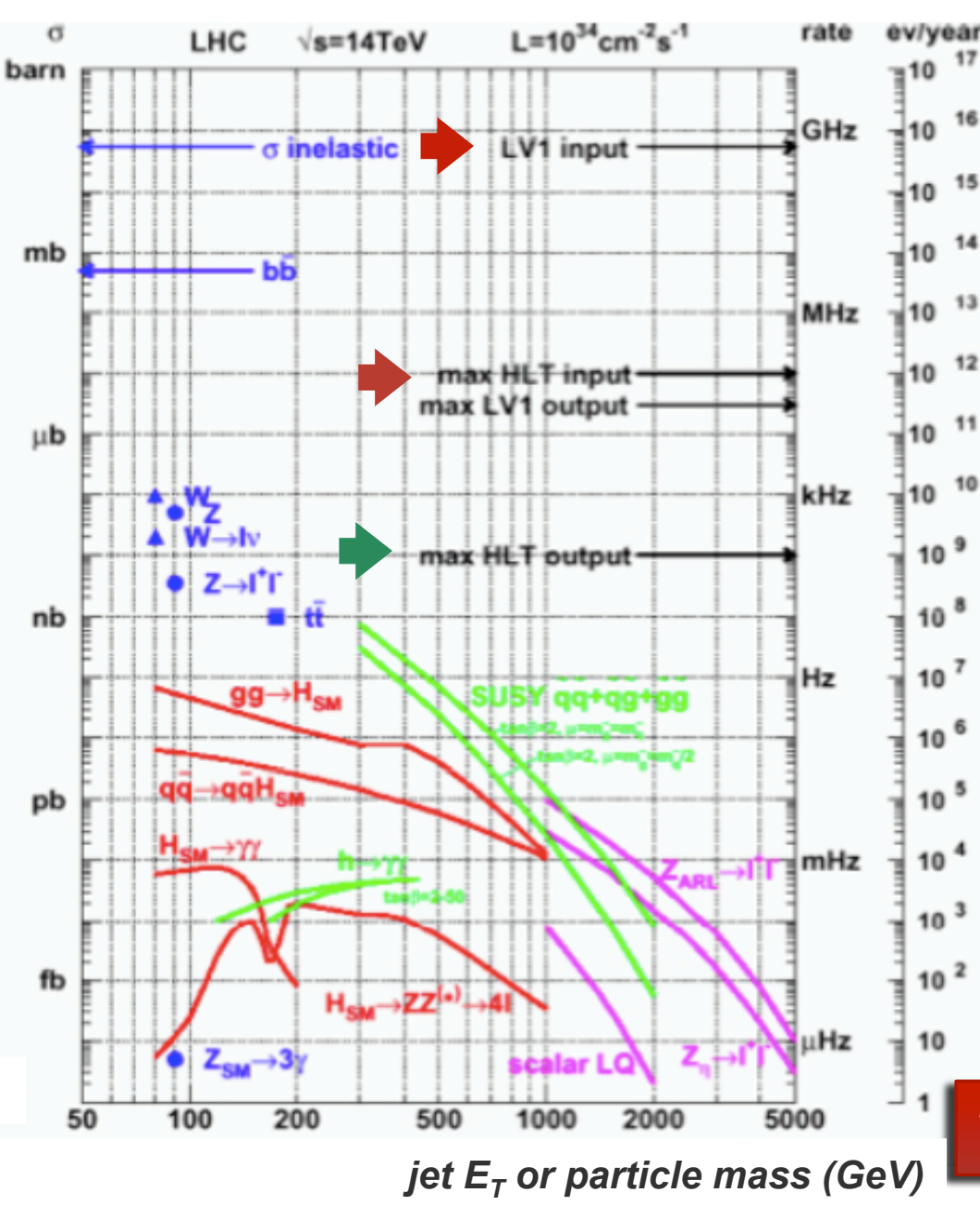

ICCSME 2009 - ATLAS trigger
2. Total non-diffractive $p p$ cross section at $\mathrm{V} s=14$ $\mathrm{TeV}$ is $\sim 70 \mathrm{mb}$

$\pi$ Collision rate: $L \times \sigma=O\left(10^{9}\right) \mathrm{Hz}$

7 Huge range of cross-sections and production rates (example with design $\mathrm{L}$ )

$$
\begin{array}{lll}
\pi & \text { Beauty }(0.7 \mathrm{mb}) & -10^{3} \mathrm{~Hz} \\
\pi & \mathrm{W} / \mathrm{Z}(200 / 60 \mathrm{nb}) & -100 \mathrm{~Hz} \\
\pi & \text { Top }(0.8 \mathrm{nb}) & -10 \mathrm{~Hz} \\
\pi & \text { Higgs - 150 GeV (30 pb) } & -0.1 \mathrm{~Hz}
\end{array}
$$

7. The output rate is limited by the offline computing budget and storage capacity $\lambda$ Only a small fraction of production rate can be used in the analysis

Trigger must reduce event rates from GHz to $200 \mathrm{~Hz}$ 

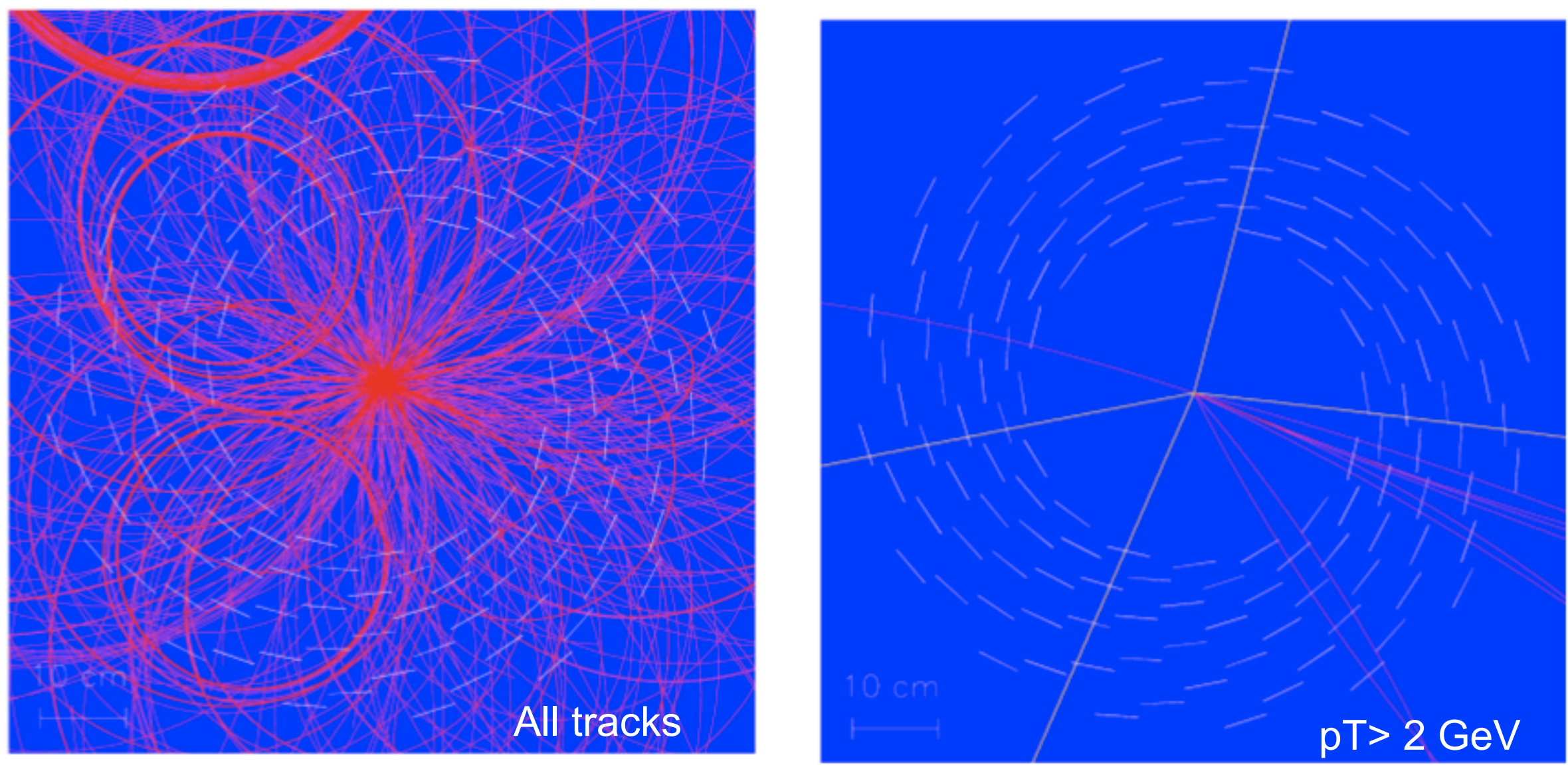

7) Average 23 interaction per Bunch Crossing at $\mathrm{L}=10^{34} \mathrm{~cm}^{-2} \mathrm{~s}^{-1}$ $\lambda$ Pile-up of interactions in the event

T) Simulated $\mathrm{H} \rightarrow 4 \mu$ event +17 minimum-bias events 


\section{Trigger signatures}

\begin{tabular}{l|l|l|}
\hline Object & Physics Measurements Examples & Trigger Chains \\
\hline electrons & Higgs, new gauge bosons, extra dim, SUSY, W, top & e25i, 2e15i \\
\hline photons & Higgs, extra dim, SUSY & $\gamma 60 \mathbf{i}, 2 \gamma 20 \mathbf{i}$ \\
\hline muons & Higgs, new gauge bosons, extra dim, SUSY, W, top & $\mu 20 \mathrm{i}, 2 \mu 10$ \\
\hline jets & SUSY, compositeness, resonances & $\mathbf{j} 400,3 \mathbf{j} 165,4 \mathrm{j} 110$ \\
\hline jet+missing $\mathrm{E}_{\mathrm{T}}$ & SUSY, leptoquarks & $\mathbf{j 7 0}+\mathbf{x E 7 0}$ \\
\hline tau+missing $\mathrm{E}_{\mathrm{T}}$ & Extended Higgs models, SUSY & $\tau 35+\mathbf{x E 4 5}$ \\
\hline
\end{tabular}

7 Selection is based on inclusive high- $\mathrm{p}_{\mathrm{T}}$ signatures, with low multiplicity (single/diobjects)

$\pi$ SM physics overlap with Tevatron and sensitive to un/predicted New Physics

7 Allow reasonable safety factors to account for physics (cross-sections, cavern background....) and detector-performance uncertainties

7. Must include triggers for monitoring and calibration/energy scale determination

$\pi$ Instrumental and physics backgrounds not completely known

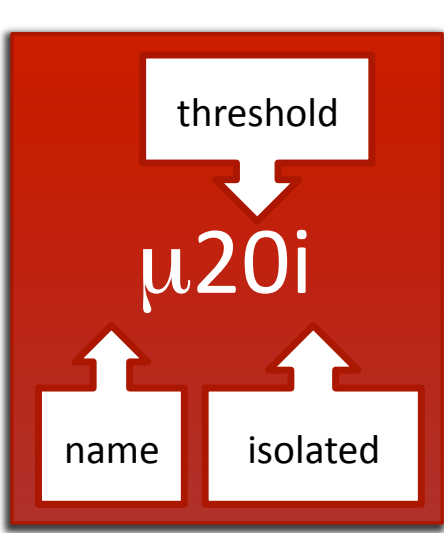

$\pi$ Detector and trigger efficiency from data 


\section{Trigger and DAQ architecture}

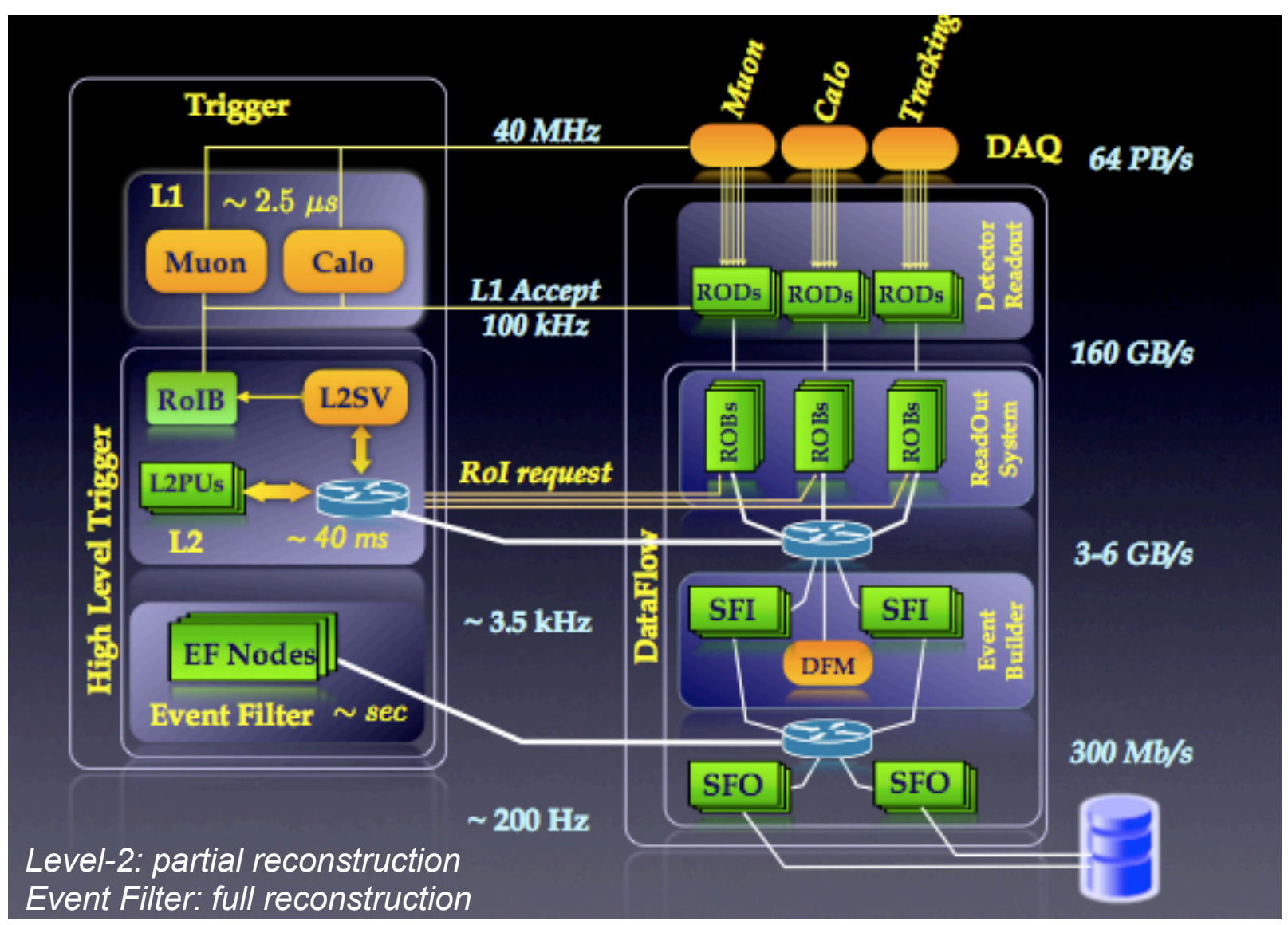

\section{7) To reduce the dataflow}

requirements, ATLAS chose a threelevel trigger architecture, exploiting the "region-of-interest" (Rol) approach

$\lambda$ Complex dataflow architecture based on local readout buffers

7 Redundancy of selection criteria leads to high trigger efficiency and the possibility to measure it from the data

$\approx$ Use of "Trigger Menus"

$\approx$ Must be sufficiently flexible to face possible variations of LHC luminosity

$\lambda$ Pile-up: event characteristics vary with luminosity and bunch structure, since the number of $p p$ interactions per bunch-crossing varies 


\section{Level-1 system}

2 Requirements

$\pi$ Rate reduction of $10^{4}$ (100 kHz output)

$\pi$ Trigger decision identifies uniquely the Bunch Crossing (25 ns)

\% Logic decisions by custom hardware

$\approx$ Data held in pipelines, with a fixed latency ( $\max 2.5 \mu \mathrm{s}$ )

₹ Fast detector response and data movement

7 Identifies physics objects from Calorimeters and Muon detectors with coarse granularity

$\pi \mathrm{EM}, \tau$, jet, $\mu, \Sigma \mathrm{E}_{\mathrm{T}}, \mathrm{E}_{\mathrm{T}}$ miss

$\pi$ Based on $E_{T}, p_{\mathrm{T}}$ (thresholds) and isolation criteria

त Central Trigger Processor

$\lambda$ Maximum 256 trigger items, combinations of one or more trigger inputs, can be deployed at any time

$\lambda$ Different pre-scale factors can be applied on each item to tune rates as luminosity varies during a fill

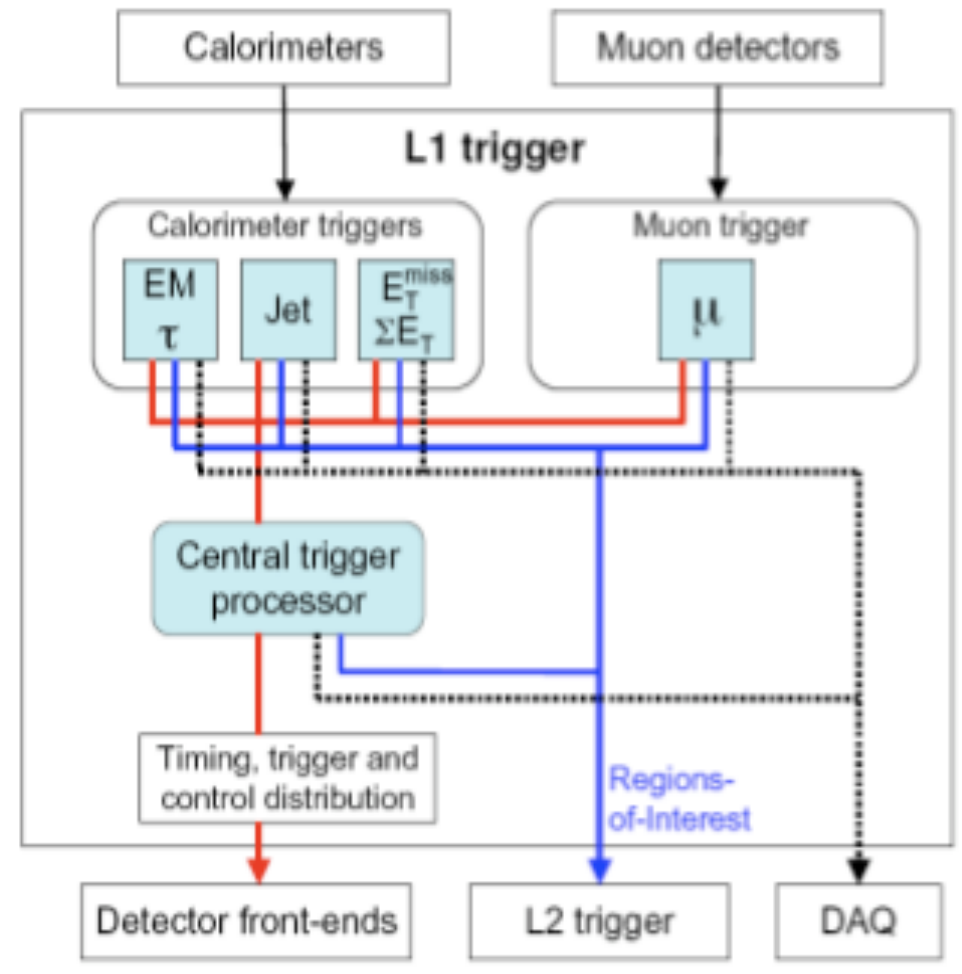

त Most important items are never pre-scaled 


\section{High Level Trigger selection}

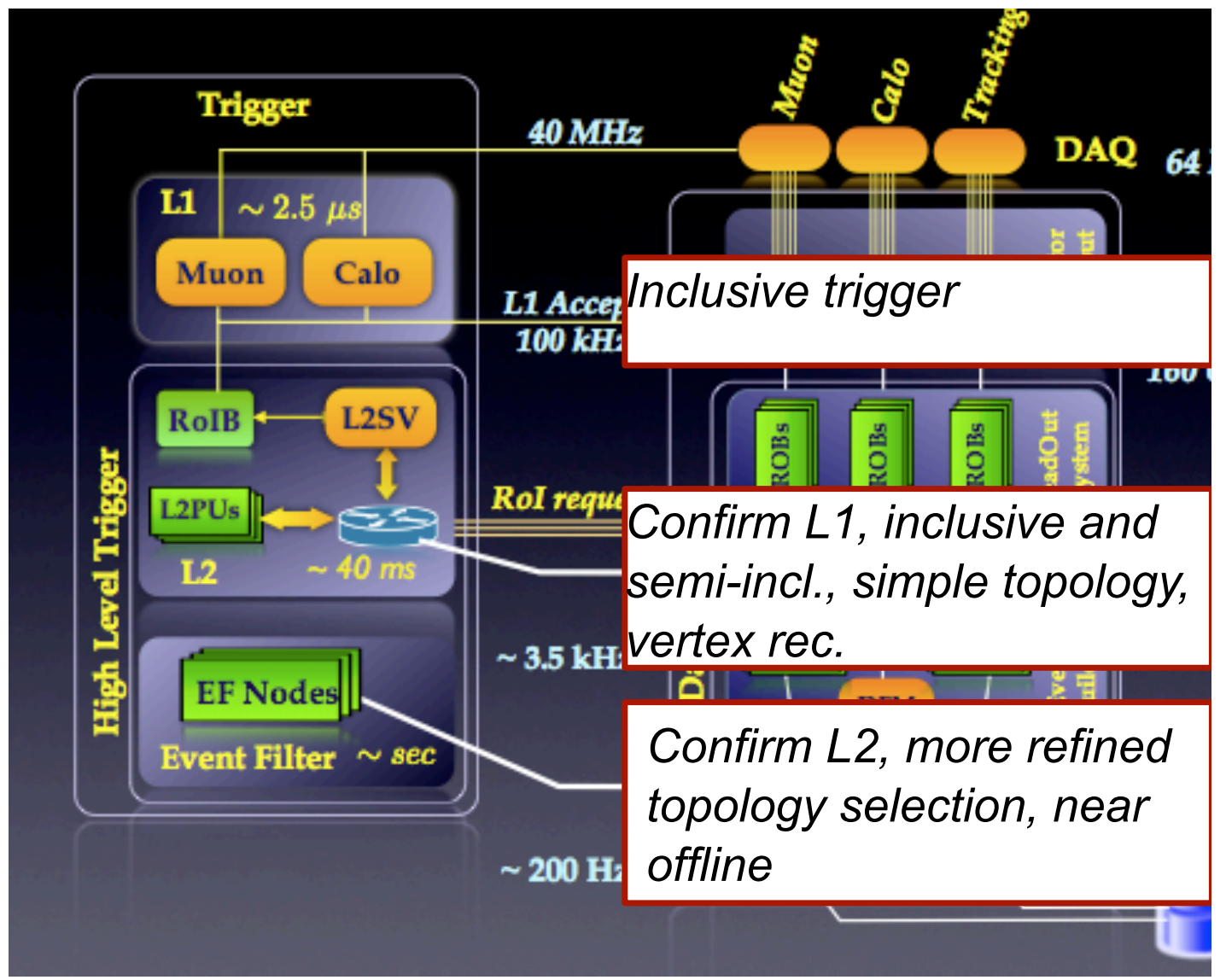

Level-2 and Event Filter (HLT) software trigger

$\lambda$ Same selection infrastructure, implemented on Linux PC farms

$\lambda$ Full granularity detector data, including tracking systems

Goal: Reduce decision latency and network traffic

7 Seeded reconstruction and early rejection

त Alternate steps of feature extraction with hypothesis testing: events can be rejected at any step with a complex algorithm scheduling

$\pi$ Regions of Interest at Level-2

$\lambda$ Custom algorithms (optimized for timing performance) selectively access and analyze data from the regions selected by L1 ( $2 \%$ of the event size)

$\lambda$ The average number of Rols per event is $\sim 1.6$ 


\section{Muon trigger design}

\section{Deviated muon found in coincidence windows}

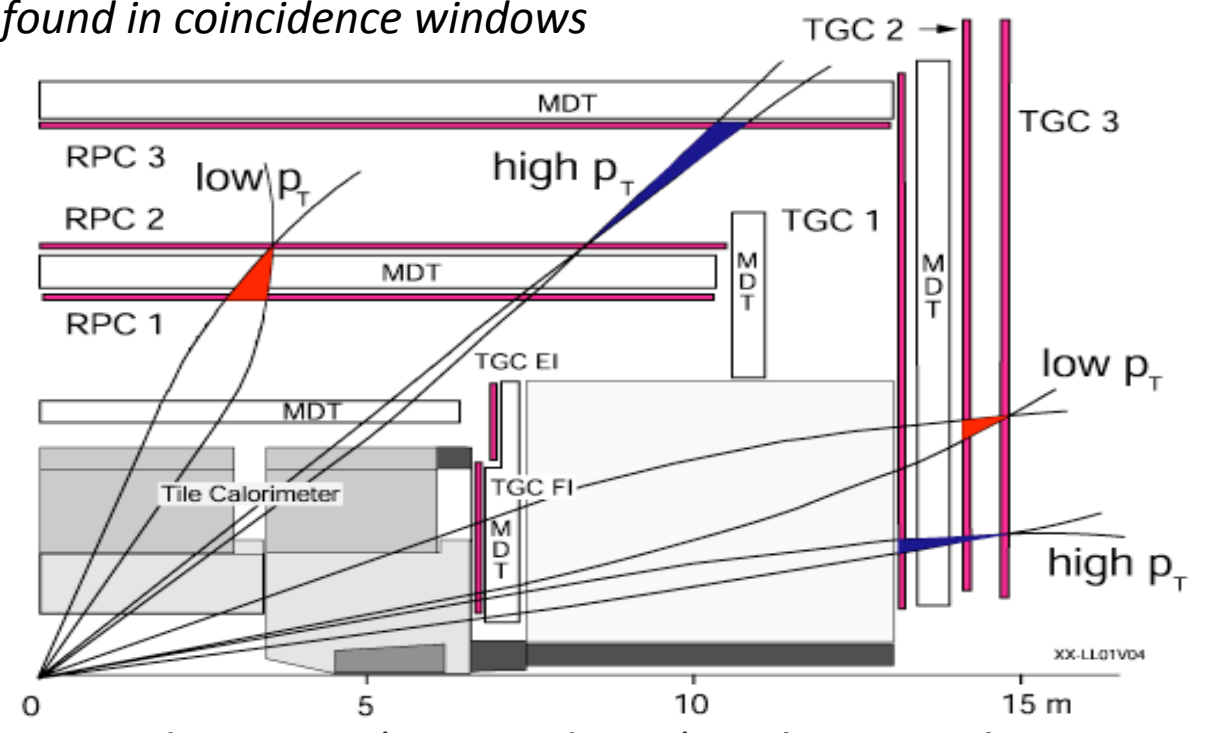

RPC time

resolution

$\sigma=1.9$ ns
Inclusive $\mu$ cross-section @ LHC (prompt $\mu$ and $\pi / K$ decay)

7 Dedicate trigger detectors (TGC and RPC) with optimal timing resolution

$\pi \quad$ Strong rejection of fake muons (induced by noise and physics background) using algorithms on 2 views (air-toroid structure)

$\pi$ Requirement for cosmic-ray and beam-halo triggers included in the design

7 Wide $\mathrm{p}_{\mathrm{T}}$-threshold range to allow B-physics studies

$\pi$ Most significant contribution to $\mathrm{L} 1$ rate is given by low- $\mathbf{p}_{\mathrm{T}}$ muons $(<3.5 \mathrm{GeV})$

7 HLT uses combined tracks in the Muon Spectrometer and the Inner

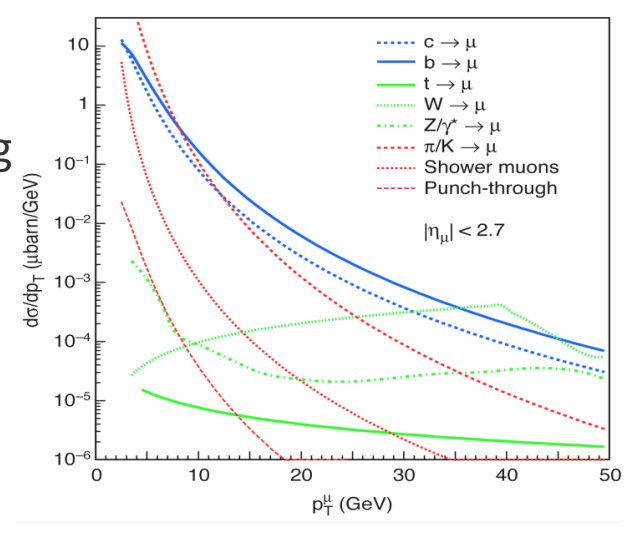
detectors and isolation criteria based on energy deposit in the calorimeters 


\section{Calorimeter trigger design}

$\geqslant$ e, $\gamma, \tau$, jets, $E_{t}$ miss $/ \Sigma E_{T}$

- $\quad$ 7200 projective trigger towers: $\Delta \eta \times \Delta \varphi=0.1 \times 0.1$

- Acceptance coverage: $\mid \eta /=4.9$ (FCAL)

$\lambda$ Various combinations of cluster sums and isolation criteria

2. Level-1

$\lambda$ Peak finder for $\mathrm{BC}$ identification

$\pi \mathrm{E}_{\mathrm{T}}$ conversion using LUT: 8-bit $\mathrm{E}_{\mathrm{T}}$, linear up to $255 \mathrm{GeV}$

$\pi$ Sliding-window technique to find clusters

$\pi$ Dedicated processors apply the algorithms, using programmable $\mathrm{E}_{\mathrm{T}}$ thresholds

त High-Level trigger

$\lambda$ More topological variables and tracking information for electrons from Inner Detectors

$\pi$ Isolation criteria can be imposed to control the rate (reducing jet background at low energies thresholds)

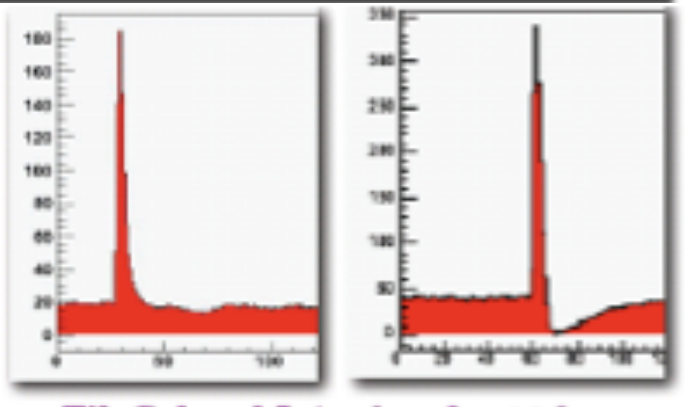

TileCal and LAr sigmals at trigger

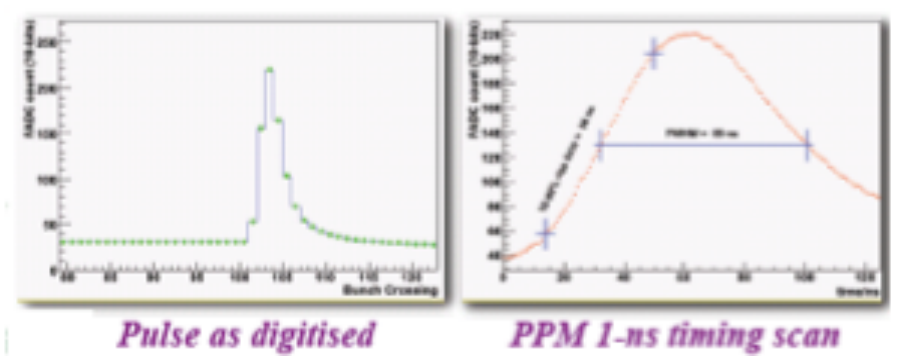

Level-1 clustering algorithm

ICCSME 2009 - ATLAS trigger

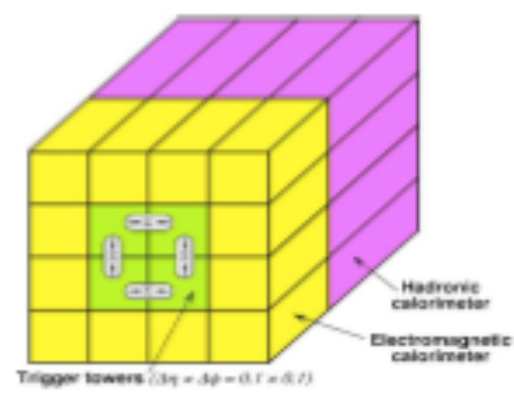

$\frac{7}{2} \quad$ Vertical sums

$\leftrightarrow \Sigma \rightarrow$ Horizontal sums

Local maximum/

Region-of-interest

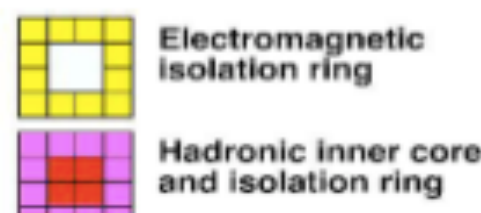

11 


\section{Expected trigger performance}

Trigger efficiency is calculated on samples of simulated signal events, with respect to the offline reconstruction

7 Described by turn-on curves whose sharpness is related to the finite $p_{T} / E_{T}$ resolution

$\lambda$ Efficiency dependency on $\mathrm{p}_{\mathrm{T}} / \mathrm{E}_{\mathrm{T}}$ and pseudo-rapidity $(\eta)$ must be minimized

\section{e10 trigger efficiency as a function of $\mathrm{E}_{\mathrm{T}}$ and $\eta$}
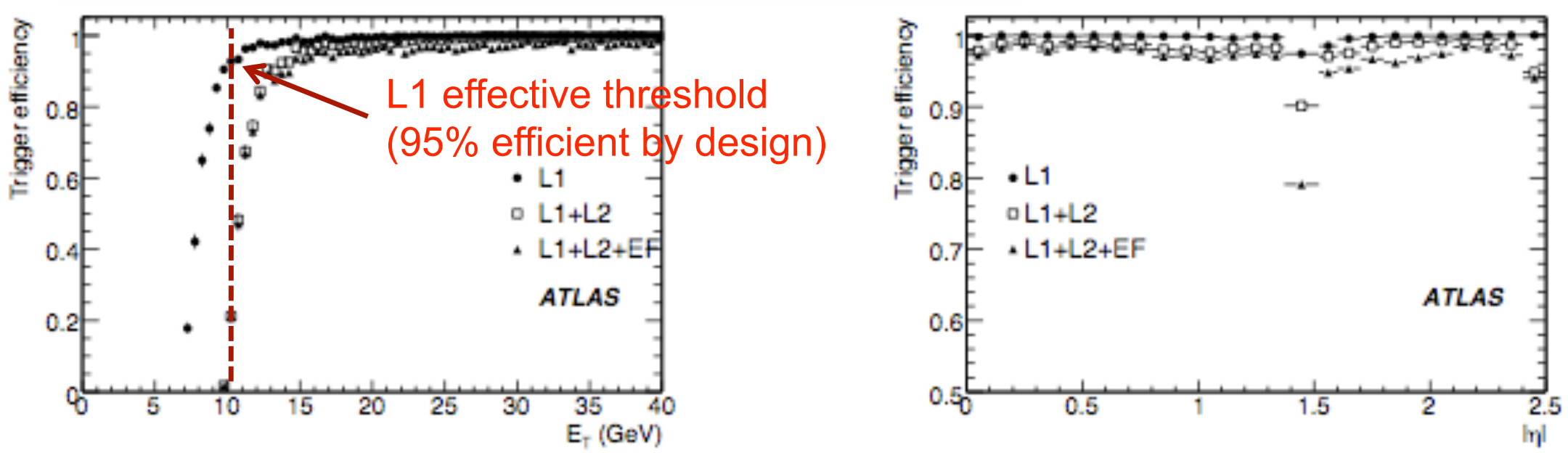


\section{Trigger efficiency measurement from data}

7 "Tag and Probe" method used where possible

$\lambda$ Clean signal sample $(\mathrm{Z}, \mathrm{J} / \psi$ to leptons)

$\lambda$ Select track that triggered the event (Tag)

$\pi$ Find the other offline track (Probe)

$\pi$ Apply trigger selection on Probe

7 The systematic uncertainties of the method are compared with Montecarlo

7 Needs deep studies on background contamination to doubla_anton cources

Muon trigger efficiency (with $1 \mathrm{fb}^{-1}$ )

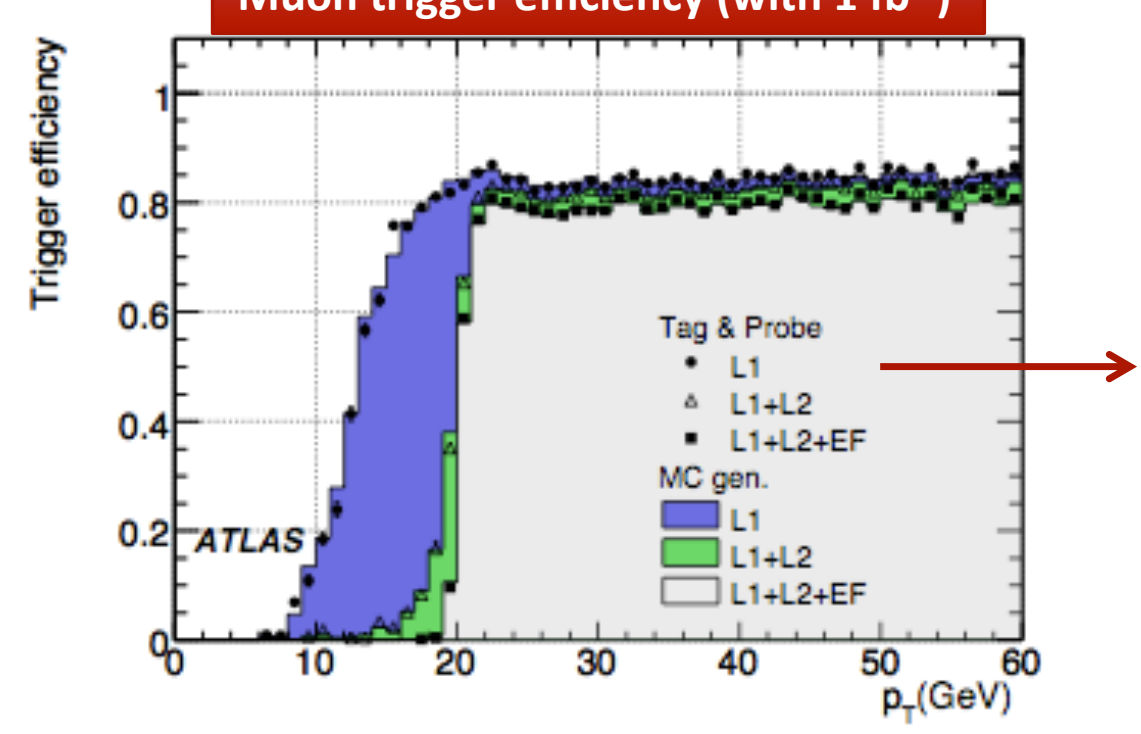

Fractional efficiency difference w.r.t. MC truth

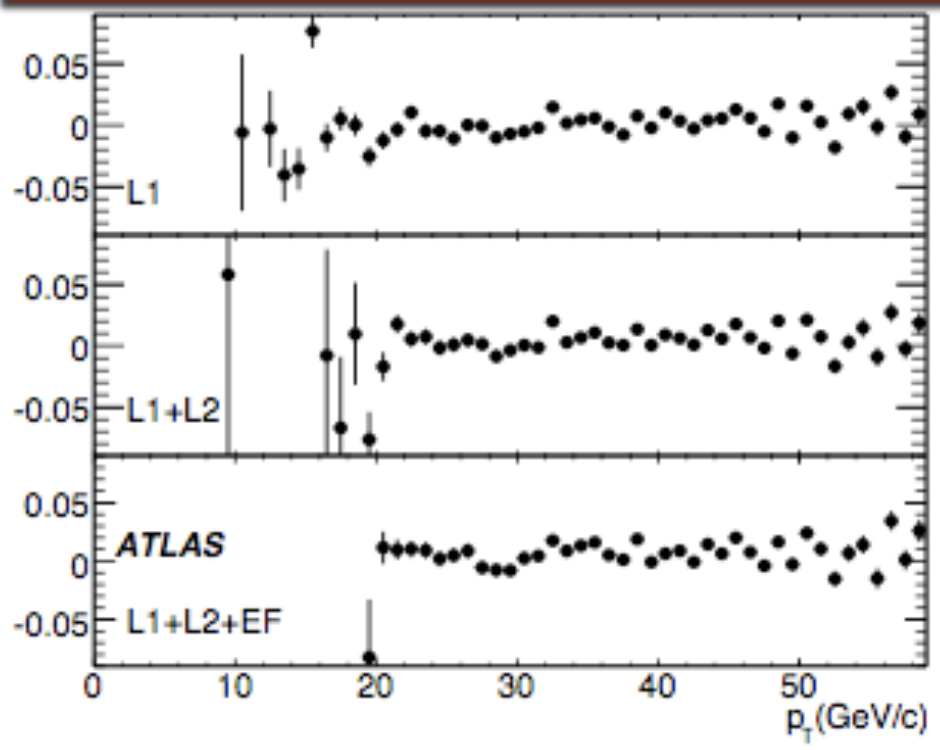




\section{Commissioning of the trigger}

7 Many competing demands on trigger for early running

$\pi$ Commissioning of Level-1 trigger and HLT

$\pi$ Detector commissioning

$\pi$ Timing-in of the detectors (readout and trigger)

$\lambda$ Commissioning of Tier-0 (reconstruction)

$\pi$ Provide calibration and alignment samples

$\lambda$ Provide samples for initial physics studies

7 Cosmic-rays runs and first experience with beams in September 2008

$\pi$ Timing-in and energy calibration of the L1 trigger

$\pi$ Test of HLT algorithms and architecture

Now

LHC start-up luminosity (up to to $10^{31} \mathrm{~cm}^{-2} \mathrm{~s}^{-1}$ )

$\pi$ Trigger and detector commissioning

$\pi$ Standard Model processes

$\lambda$ Selection strategy focused on

$\pi$ Low thresholds and loose selection criteria

HLT algorithms in pass-through mode (only at the very beginning) 


\section{Status of the trigger architecture}

Level-1 trigger

$\lambda$ Tested in readout up to $80 \mathrm{kHz}$

$\pi$ Timing-in in progress

$\geqslant$ High-Level triggers

त $850 \mathrm{HLT}$ nodes (35\% final system) tested at $60 \mathrm{kHz}$

त Finalization of the system will be luminosity driven Expected: 500 Level-2 nodes +1800 Event-Filter nodes (8 cores @ $2.5 \mathrm{GHz}, 2$ GB Memory/node )

$\pi$ Stress-test system with $10^{31} \mathrm{~cm}^{-2} \mathrm{~s}^{-1}$ trigger menu during Technical Runs

$\approx$ Simulated raw data preloaded into the readout systems and played back through the HLT/DAQ system

7 DAQ routinely running since 2008

$\lambda$ All ROS PCs installed and working

$\pi$ Dataflow networking infrastructure fully in place

$\pi$ Local storage farm (SFO) of 5 nodes with a total storage area of $50 \mathrm{~TB}$

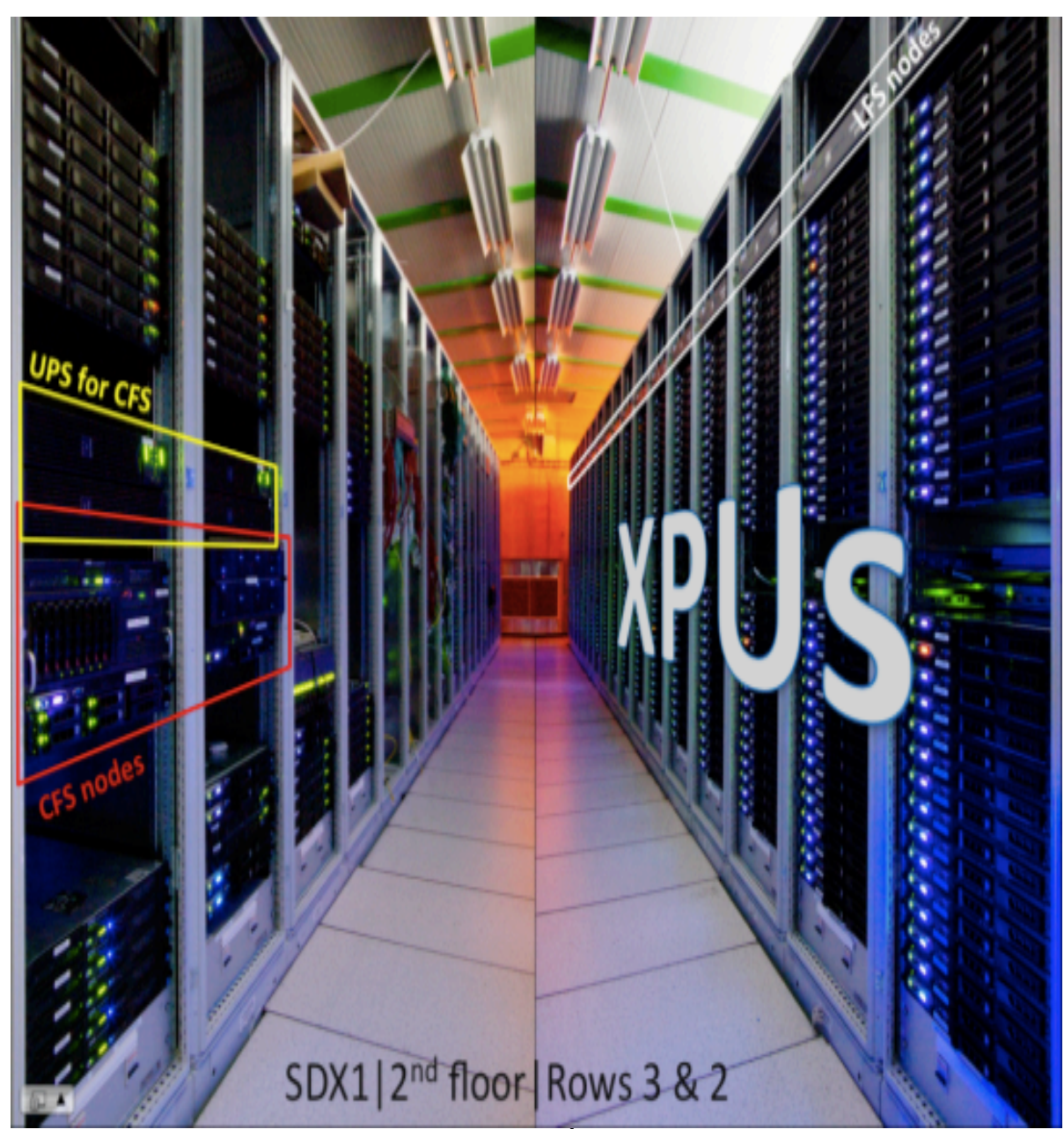




\section{First beams in ATLAS}

7 On September $10^{\text {th }} 2008$ first LHC beams reached ATLAS detector

$\pi$ Single beams initially with no RF

$\lambda$ Series of splash events generated by dumping beams on closed collimators $140 \mathrm{~m}$ from ATLAS

$\lambda$ Then circulating beams

7 ATLAS was ready

$\pi$ All the trigger infrastructure was running (HLT available)

2) Crucial test for the L1 trigger

$\lambda$ Test of triggers dedicated to detect the beams

$\pi$ Time alignment 


\section{Detecting beams}

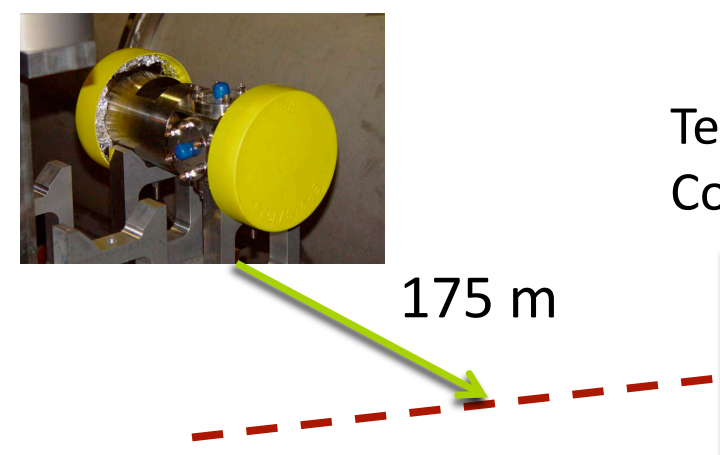

Beam Pick-up

Timing Experiment (BPTX)

$\geqslant$ Provides a filled-bunch trigger

7. Monitor the beam activity

$\lambda$ Identifies the bunches in the beams

$\lambda$ Measures their individual intensity and phase relative to the LHC clock, assigning the Bunch crossing

$\pi$ Monitor of timing signals

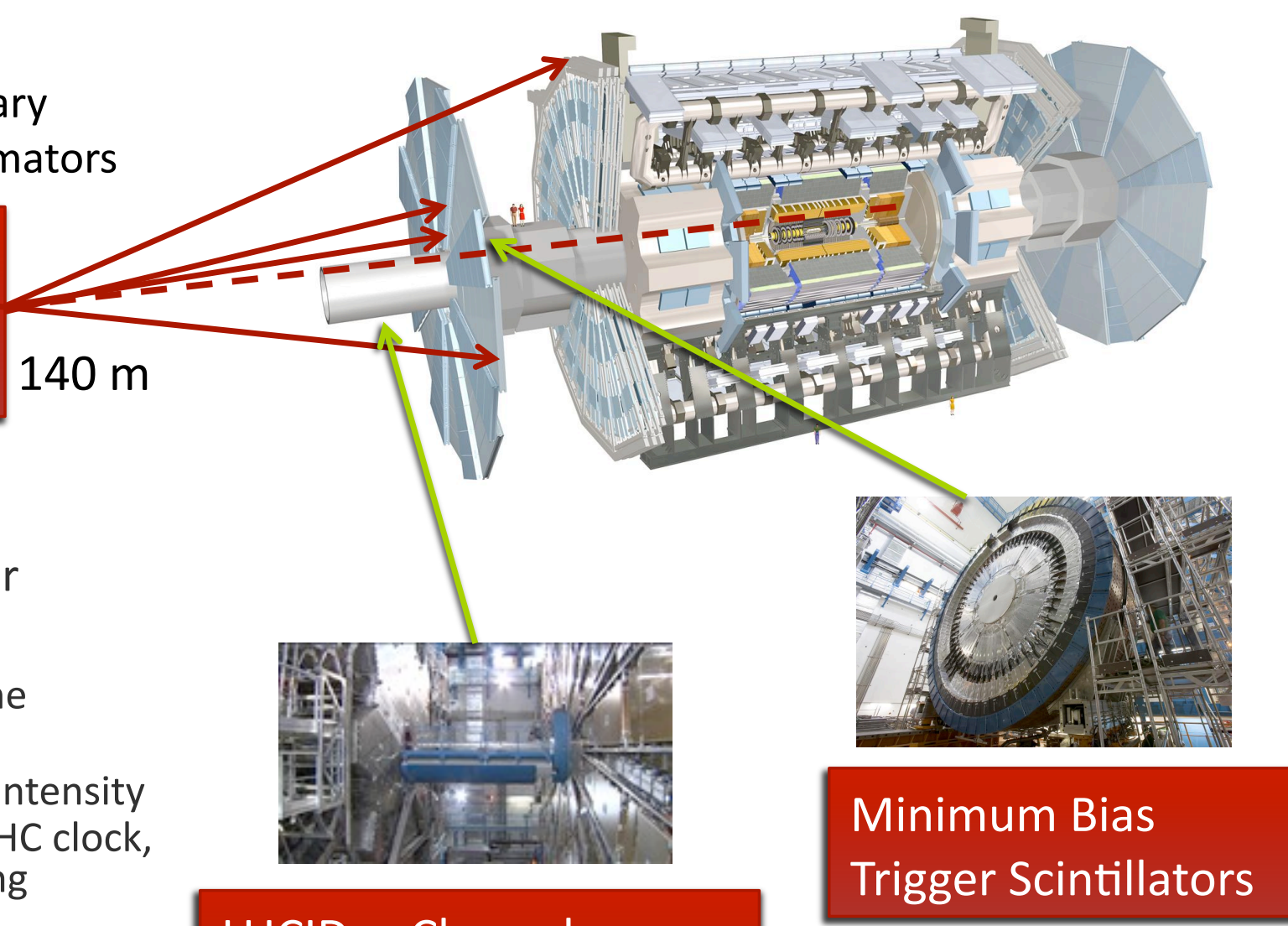

LUCID, a Cherenkov

Luminosity monitoring 


\section{Beams on $10^{\text {th }}-12^{\text {th }}$ September 2008}

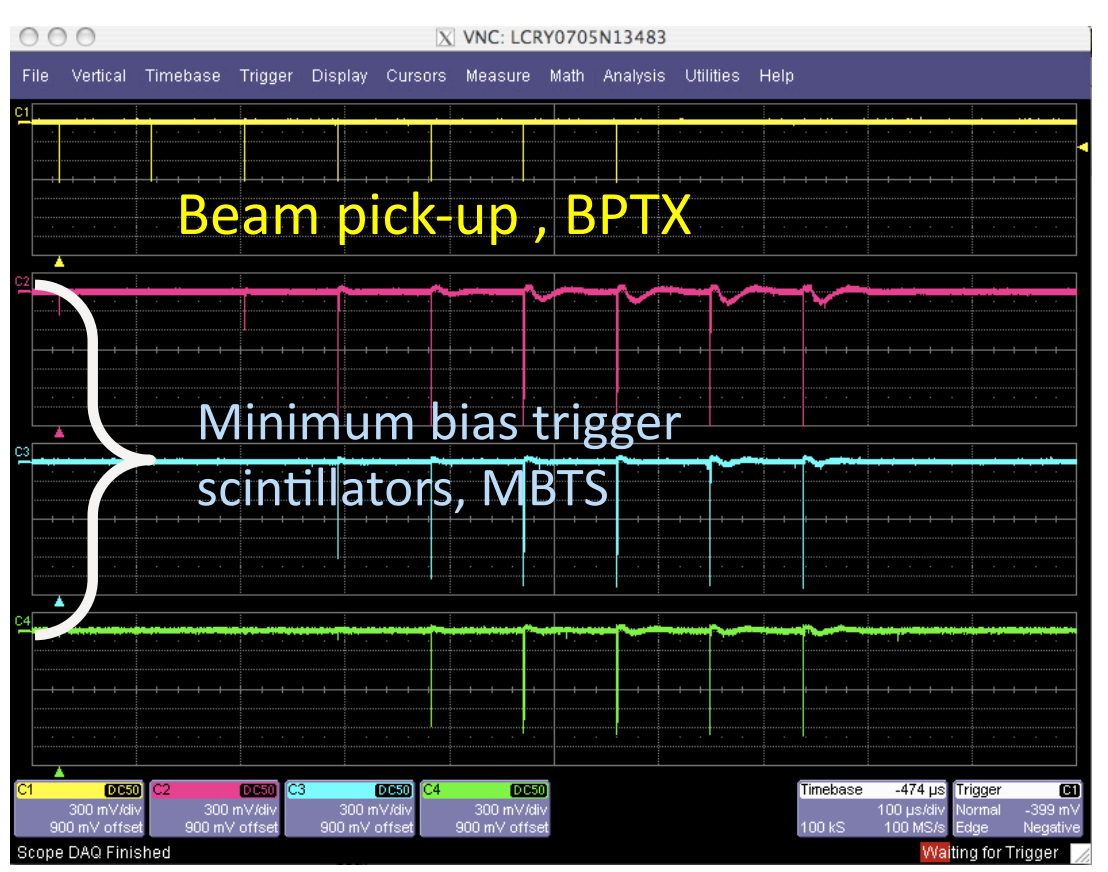

- Oscilloscope traces of discriminated signals during an injection of 1 bunch without RF capture

$\pi$ The bunch circulated a few times

$\lambda$ After 7 turns its intensity falls below the threshold of the BPTX discriminator.

$\pi$ The first few turns give only small activity in the MBTS, while after 3 or 4 turns they show saturated signals

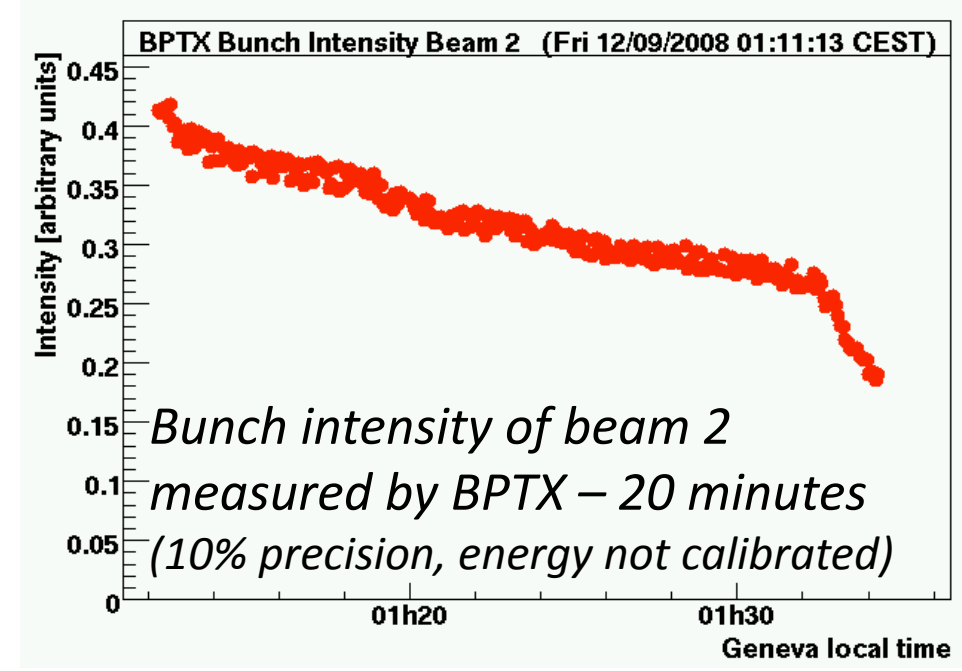




\section{The first event - triggered with cosmic settings}

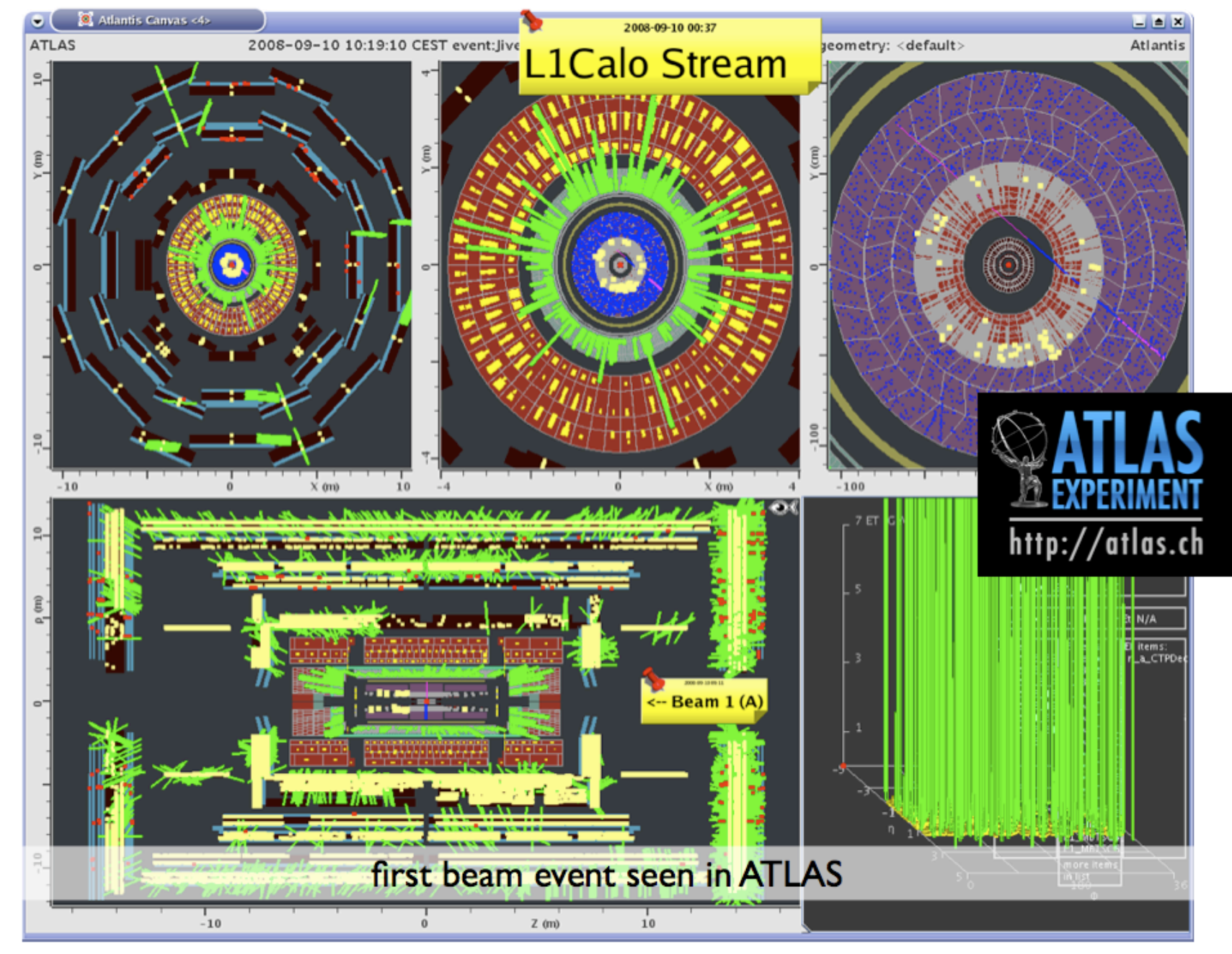




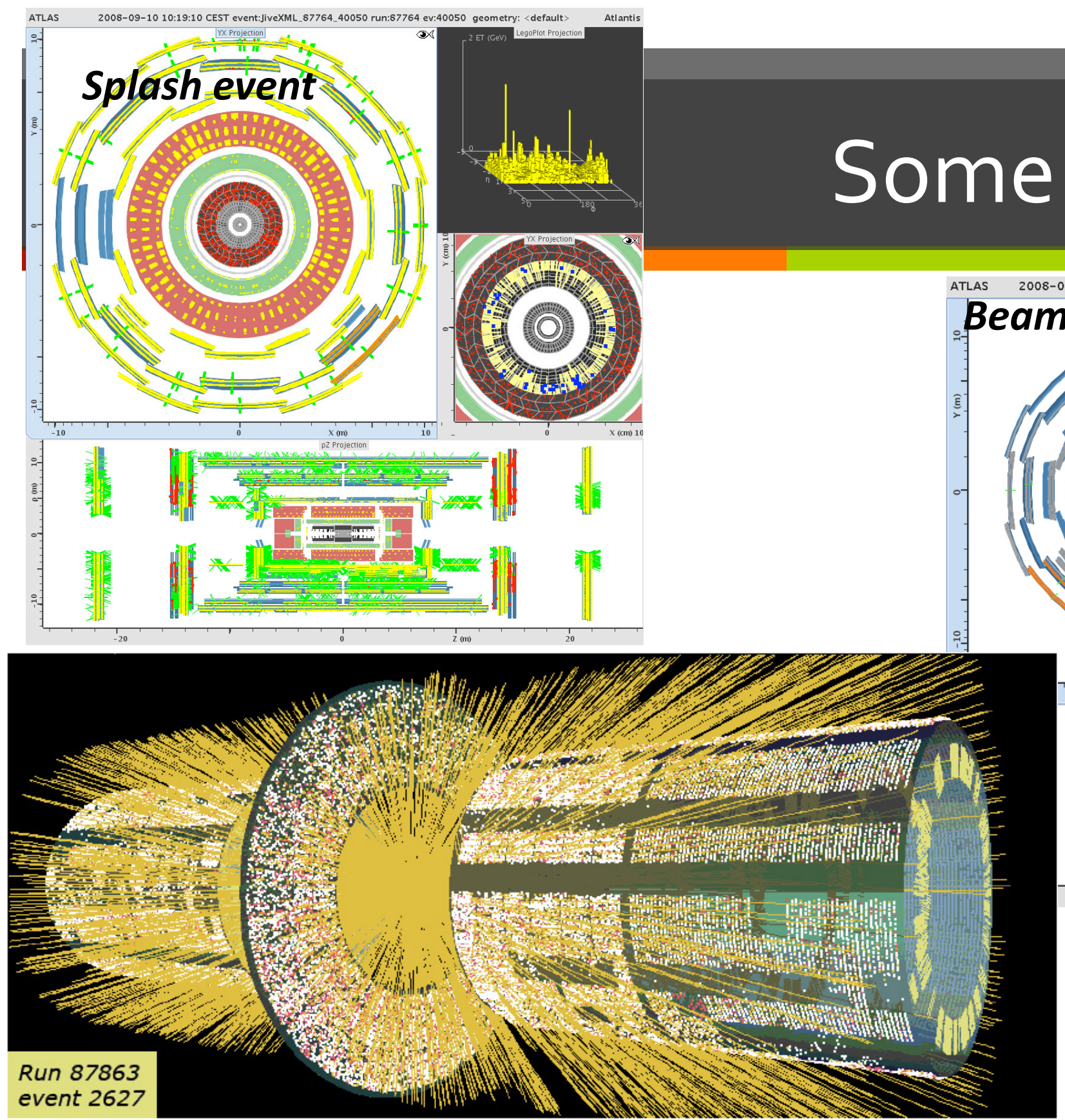

ICCSIME 2009 - ATLAS trigger 


\section{Timing-in the L1 systems with beams}

7 Exploiting the excellent timing of BPTX and MBTS, much progress on timing set-up over two days

त With circulating beams BPTX was pre-scaled and trigger relied on combinations with MBTS and calorimeter

$\pi$ Detectors worked on adjusting readout timing in parallel

$\checkmark$ With single beam, downstream timing similar to that for collisions

$\checkmark$ L1 timing-in is going on with cosmic events,

$\checkmark$ Precise timing adjustment will need collision data

Timing distribution of $L 1$ triggers from the first and third days of single-beam data. Reference triggers have $B C=0$ (note change of scale and log vertical scale)
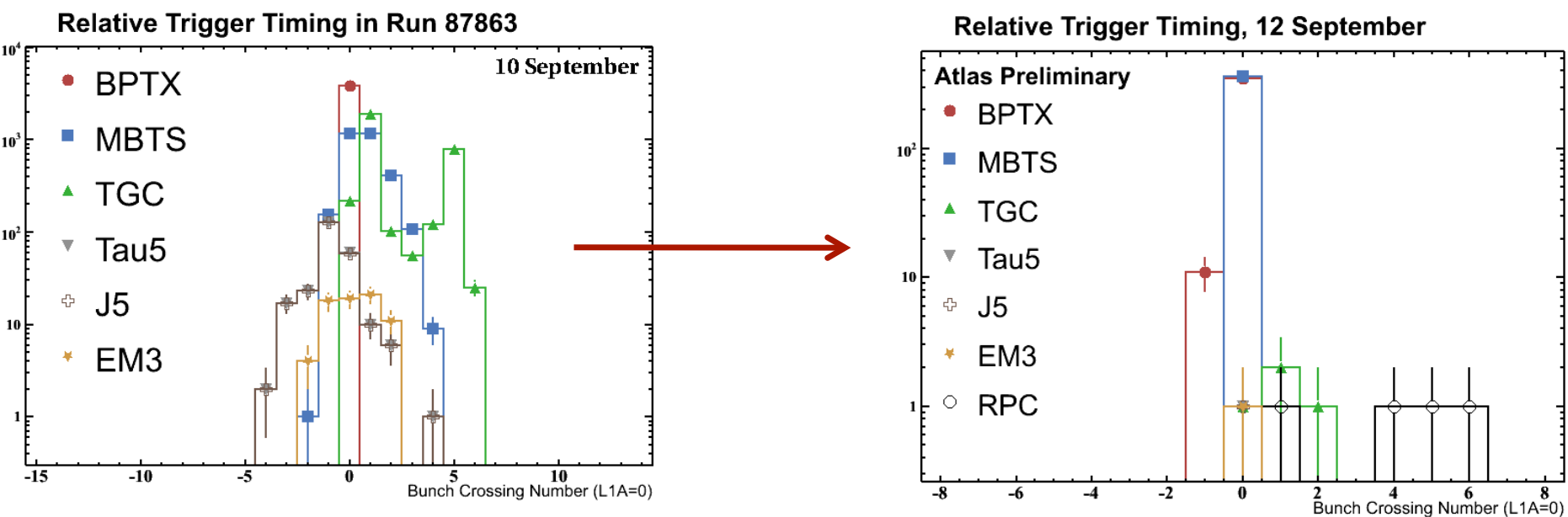


\section{L1 trigger commissioning}

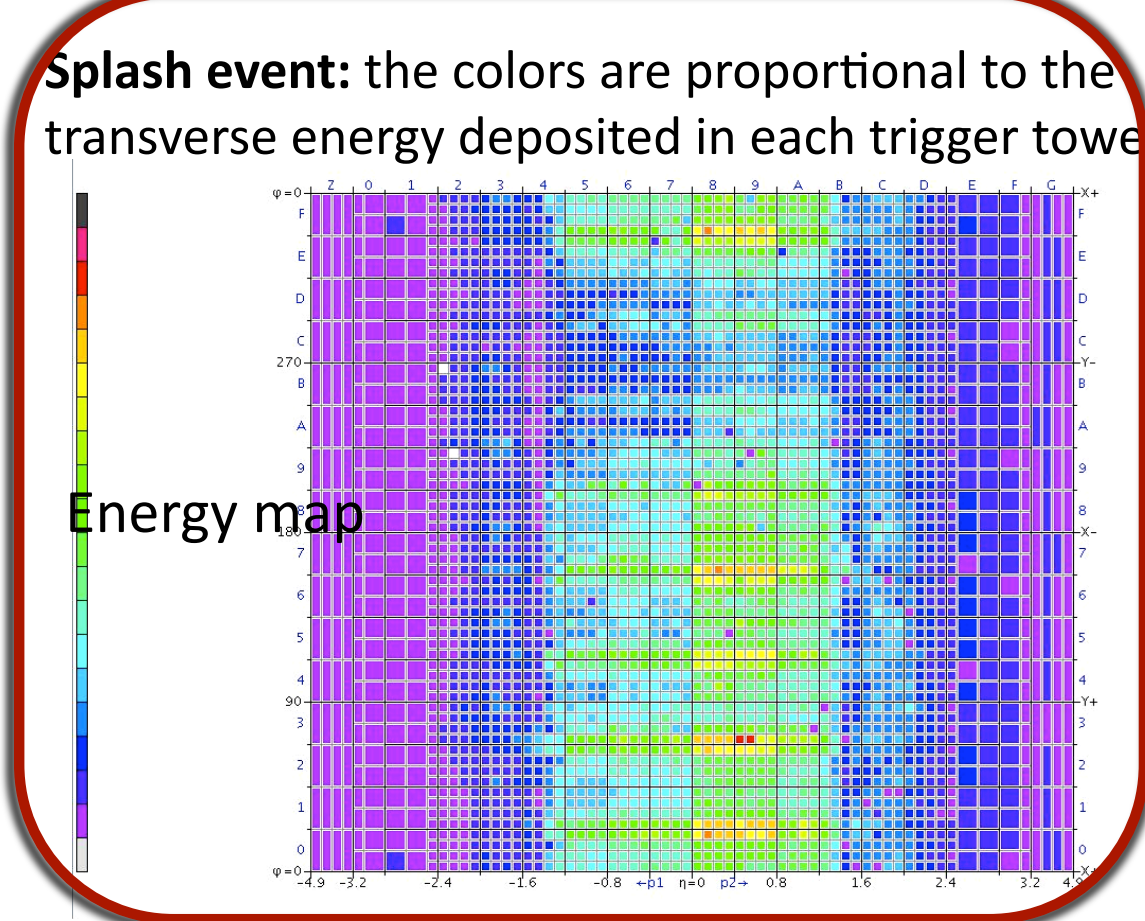

7. Cosmic rays used to check timing and calibrate $\mathrm{E}_{\mathrm{T}}$ and $\mathrm{p}_{\mathrm{T}}$ thresholds (or validate procedure done with pulses and sources)

RPC timing alignment: $R M S=5.5 n s$

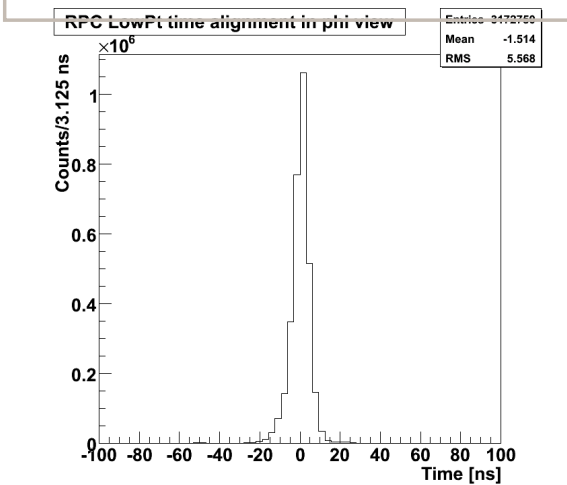

TGC: more than 99\% of the triggers within the correct $B C$

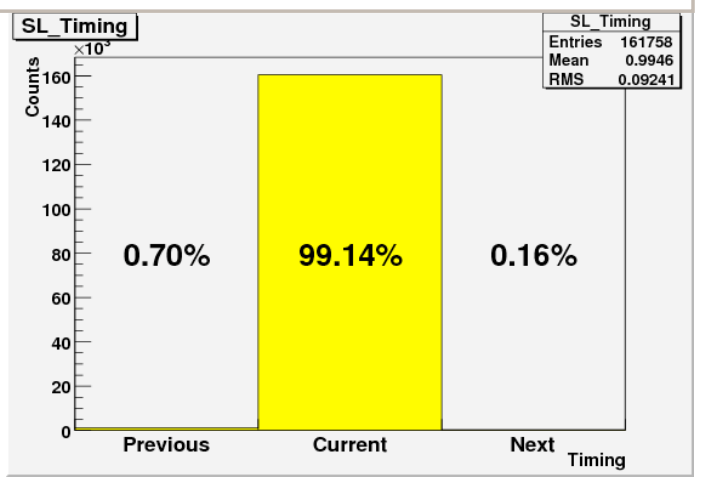

Hadronic Trigger Tower

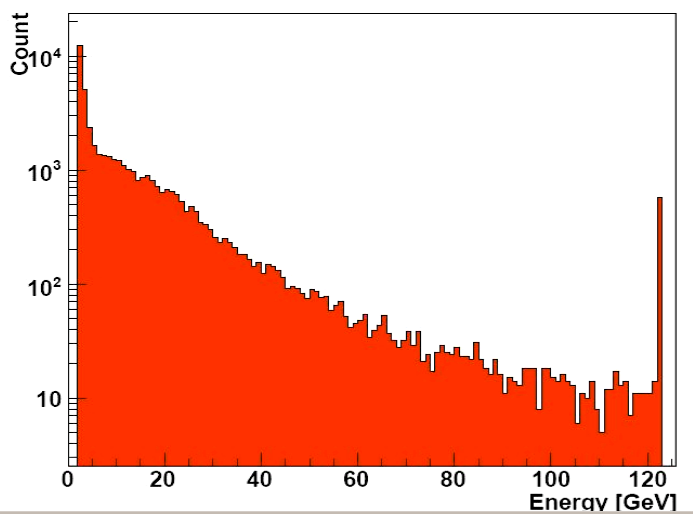

Cosmic $E_{T}$ spectrum: large tail of higher energies mainly due to large showers 


\section{HLT performance with cosmic rays}

7. More than 200 trigger items tested online

7 Level-2 processing time is compatible with the design (40 ms)

$\lambda$ The HLT acceptance/rejection is consistent with offline running

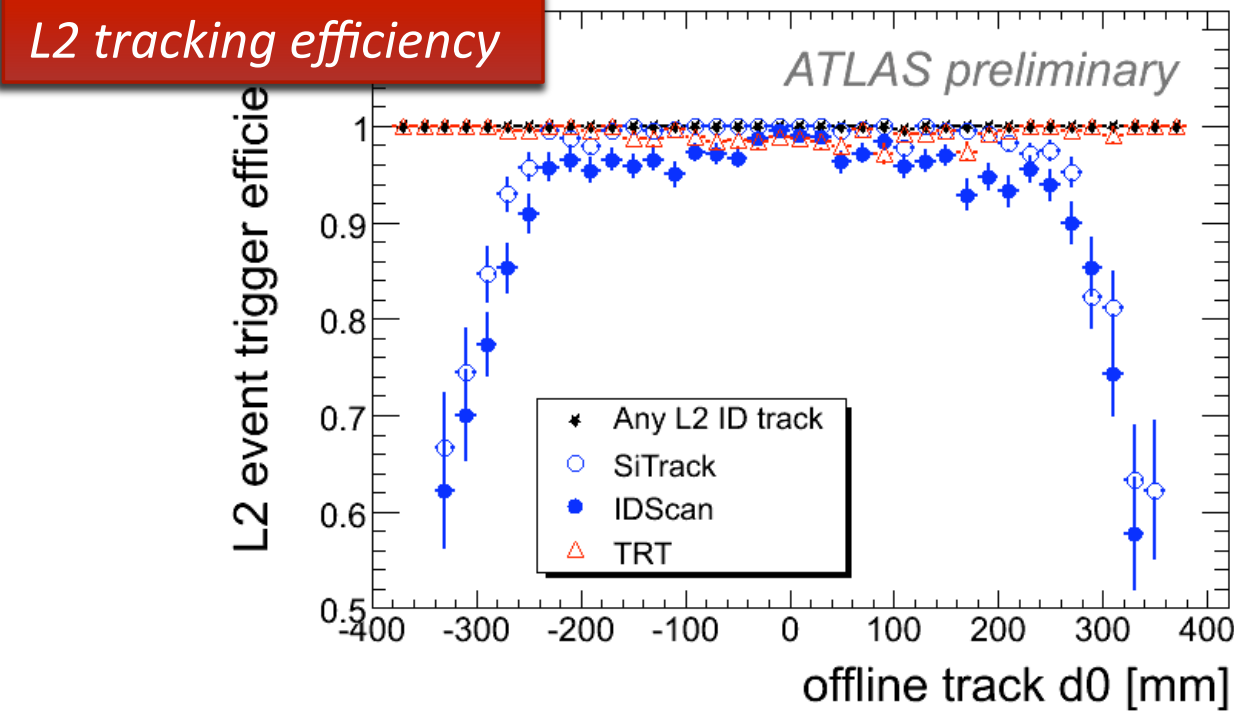

shower shape used for electron and

photon selections

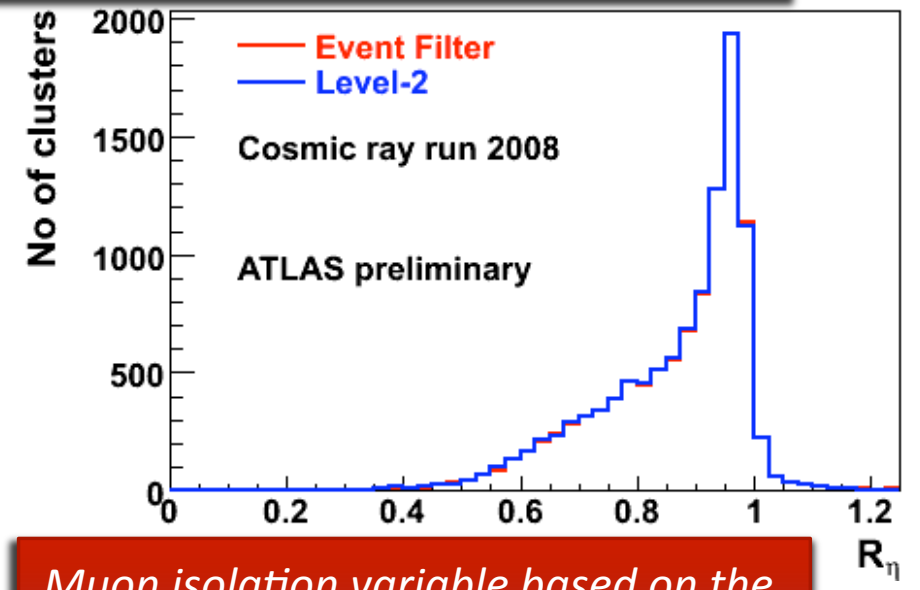

Muon isolation variable based on the Inner Detector (ID)

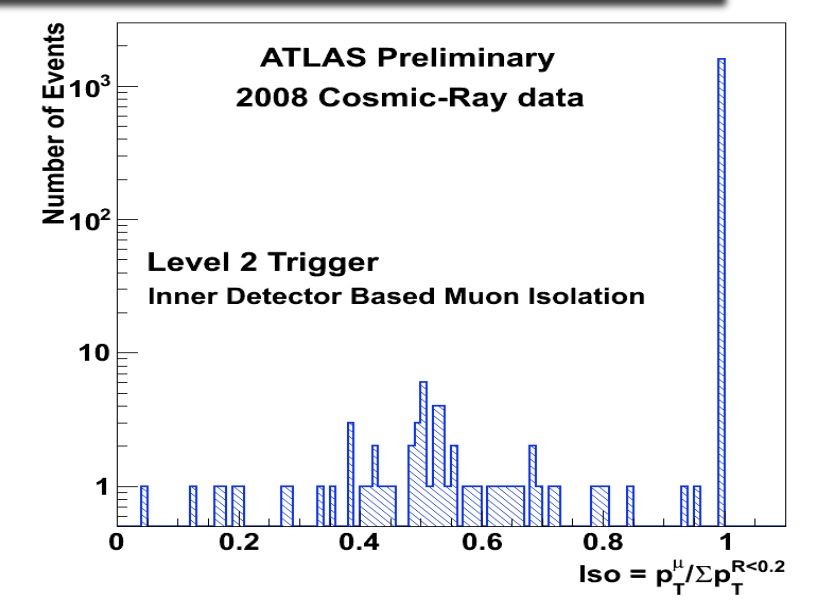




\section{Trigger rates allocation}

$\checkmark$ Target is Event Filter output rate $=200 \mathrm{~Hz}$

$\checkmark$ Trigger rate allocation on each trigger item based on

$\checkmark$ Physics goals (plus calibration, monitoring samples)

$\checkmark$ Required efficiency and background rejection

$\checkmark$ Bandwidth consumed

7 Trigger rates are calculated from large samples of simulated data, including large cross-section backgrounds (minimum-bias and QCD)

$\pi \quad$ Large samples of background events are required (7 million non-diffractive events @ 70mb used as minimum-bias sample for $10^{31} \mathrm{~cm}^{-2} \mathrm{~s}^{-1} \mathrm{menu}$ )

$$
R_{i}=\mathrm{L} \int_{p_{T-i n f}}^{p_{T-\text { cutoff }}} \frac{d \sigma_{i}}{d p_{T}} \varepsilon\left(p_{T}\right) d p_{T} \begin{gathered}
\text { Trigger } \\
\text { Efficiency }
\end{gathered}
$$

$\pi$ Given by convolution, over a given $\mathrm{E}_{\mathrm{T}} / \mathrm{p}_{\mathrm{T}}$ range, of the estimated efficiency with the cross sections representing the main trigger source

7 Large uncertainties due to detector response and jet cross-sections

$\lambda$ To be tuned with early data 


\section{Expected trigger rates @ start-up $L=10^{31} \mathrm{~cm}^{-2} \mathrm{~s}^{-1}$}
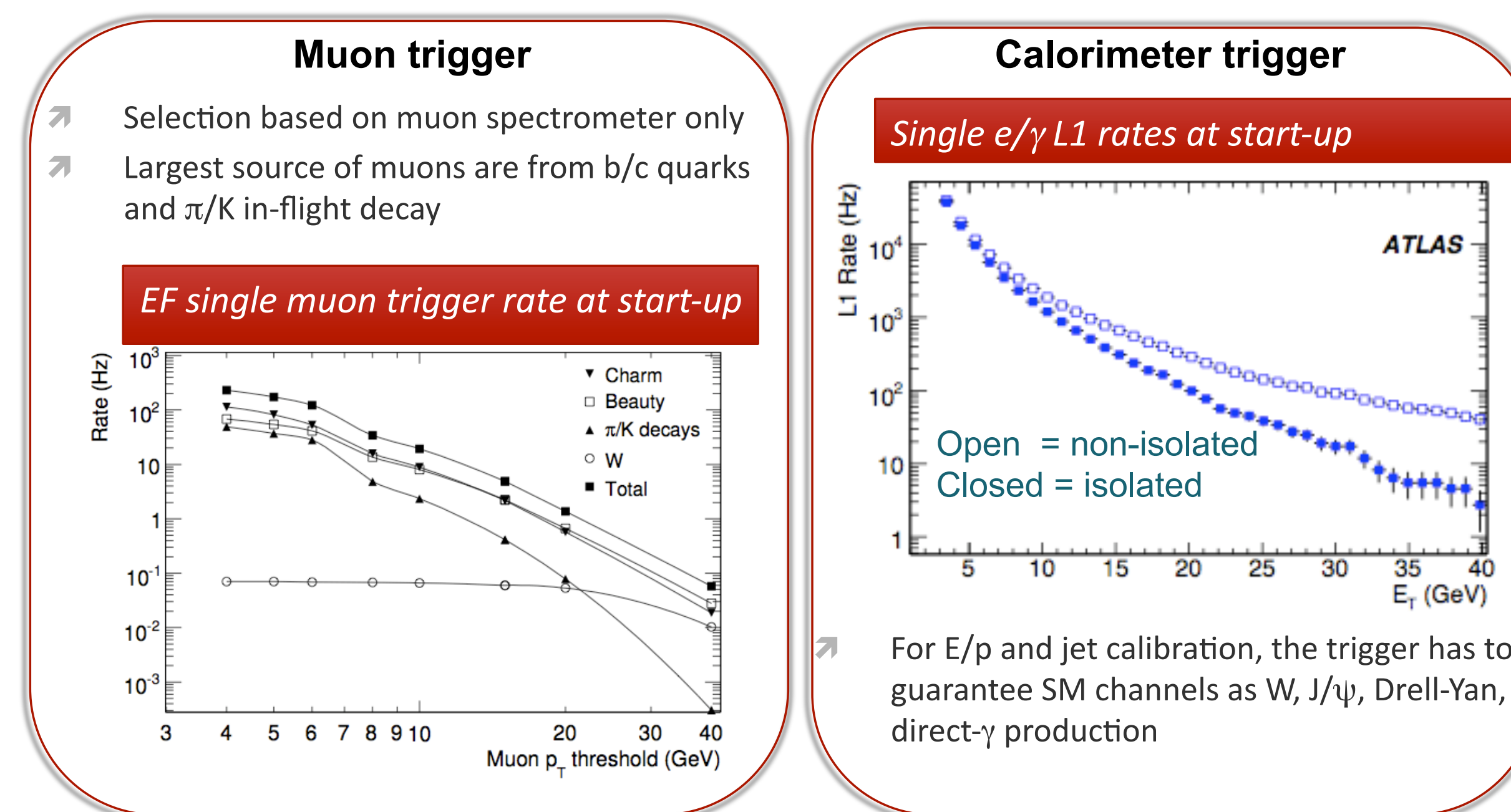


\section{Running strategy}

2) At start-up $\mathrm{L}=10^{31} \mathrm{~cm}^{-2} \mathrm{~s}^{-1}$

$\pi \quad$ L1: low $p_{\mathrm{T}}$ thresholds and loose selection criteria (adding pre-scales to control rates)

$\pi \quad$ L1: deploy high thresholds and multiobjects triggers for validation (to be used as back-up triggers)

7 HLT: running in pass-through mode for offline validation or with low thresholds

7 As LHC luminosity reaches the design, complex signatures and higher $\mathrm{p}_{\mathrm{T}}$ thresholds will be necessary to reach the physics goals

$\checkmark$ Start-up trigger Menu contains $\sim 130$ Level-1 items and $\sim 180$ HLT selection chains

$\checkmark$ e/ $\gamma$ and muon triggers are unprescaled, except EM3

\begin{tabular}{|c|c|c|c|}
\hline Object & L1 (Hz) & L2 (Hz) & $\mathrm{EF}(\mathrm{Hz})$ \\
\hline Single-electrc & 5580 & 176 & 27.3 \\
\hline Multi-electron & 649 & 41.1 & 6.9 \\
\hline Multi-photons & common & 2.9 & $<0.1$ \\
\hline Single-photons & common & 33.4 & 9.1 \\
\hline Multi-Jets & 221 & 7.9 & 7.9 \\
\hline Single-Jets & 24.4 & 24.4 & 24.4 \\
\hline Multi-Fjets & 2.7 & 2.7 & 2.7 \\
\hline Single-Fjets & 3.7 & 3.7 & 3.7 \\
\hline Multi-bjets & common & 12.9 & 2.6 \\
\hline Single-bjets & common & 11.6 & 11.6 \\
\hline Multi-taus & 465 & 14.5 & 12.4 \\
\hline Single-taus & 148 & 32.9 & 22.3 \\
\hline Multi-muons & 68.6 & 5.8 & 2.3 \\
\hline Single-muons & 1730 & 204 & 21.8 \\
\hline Missing $E_{T}$ & 57.9 & 31. & 3.8 \\
\hline Total $E_{T}$ & 6.3 & 6.3 & 1 \\
\hline Total Jet $E_{T}$ & 1.6 & 1.6 & 1.6 \\
\hline BPhysics & common & 25 & 13 \\
\hline Muti-Object & 5890 & 134 & 48 \\
\hline Minimum Bias & 1000 & 10 & 10 \\
\hline Total & 12000 & 620 & 197 \\
\hline
\end{tabular}

Example of possible sharing of bandwidth 


\section{Concluding remarks}

7 The preparations for commissioning with beam are well advanced

7 Considerable progress made in short period with single beams last year

$\lambda$ First steps made in timing set-up

$\pi$ Much useful experience gained - improved tools, procedures, etc

7 Partitioning of the trigger architecture allows commissioning in parallel

$\lambda$ Detectors can work in parallel on different partitions

$\lambda$ Events that are selected are sent, inclusively, to one or several "streams", providing samples enriched in events relevant for commissioning.

2 Trigger menus take into account the needs of the detectors

त Calibration, alignment, commissioning

7. Much effort to ensure flexibility and ability to react quickly

$\pi$ E.g. triggers to address ad hoc needs for detector commissioning 
Back-up slides 


\section{Data acquisition performance tests}

7 Dedicated Technical Runs to establish performance and scalability during system deployment

$\pi$ Simulated data as input (background + signal)

7 Test with $\mathrm{L}=10^{31}$ trigger menu

$770 \%$ of $L 2,20 \%$ of $E F$

7 Small event size: $800 \mathrm{kB}$, expected is $1.6 \mathrm{MB}$

2. Stress test for the Data Acquisition system

ROS request EB and $L V L 2$ Rates $(\mathrm{Hz})$ during ATLAS combined cosmic run

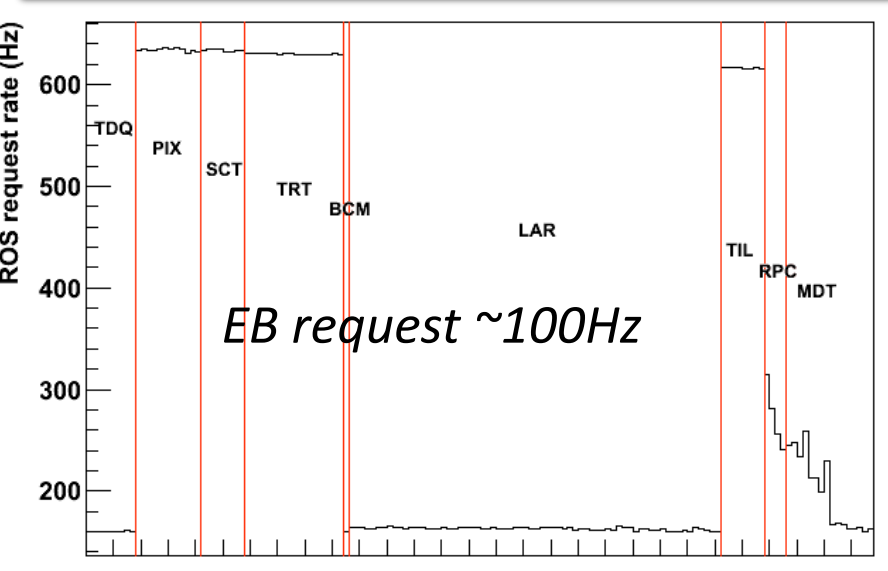

7. Readout system (ROS) request rate up to $30 \mathrm{kHz}$ 7 Maximum expected is $20 \mathrm{kHz}$ (20\% of L1)

₹ Event Building rate (L2-driven) : $4.2 \mathrm{kHz}(3.5 \mathrm{kHz}$ by design)

7 Data storage (SFO) I/O rate: $550 \mathrm{MB} / \mathrm{s}$, with peak > 700 $\mathrm{MB} / \mathrm{s}$

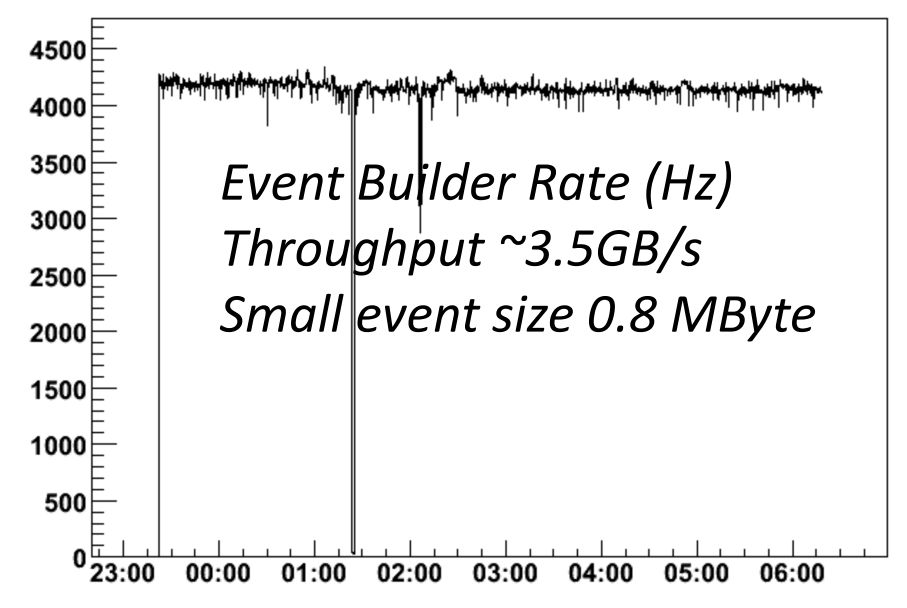




\section{Muon trigger performance}

7 Use of Inner Detector for muon $\mathrm{p}_{\mathrm{T}}<50 \mathrm{GeV}$ tracks helps:

$\pi \quad$ L2 low rejection at low-pt due to large scattering angles in the Muon Spectrometer

$\pi \mathrm{p}_{\mathrm{T}}$ resolution in regions of inhomogeneous magnetic field

$\pi$ Rejection of muons from $\mathrm{K} / \pi$ decays (small kink)

Efficiency determination validated on $\mathrm{Z}$ and $\mathrm{J} / \psi$ decays

Muon trigger efficiency and fractional efficiency for each trigger level with $50 \mathrm{pb}^{-1}$
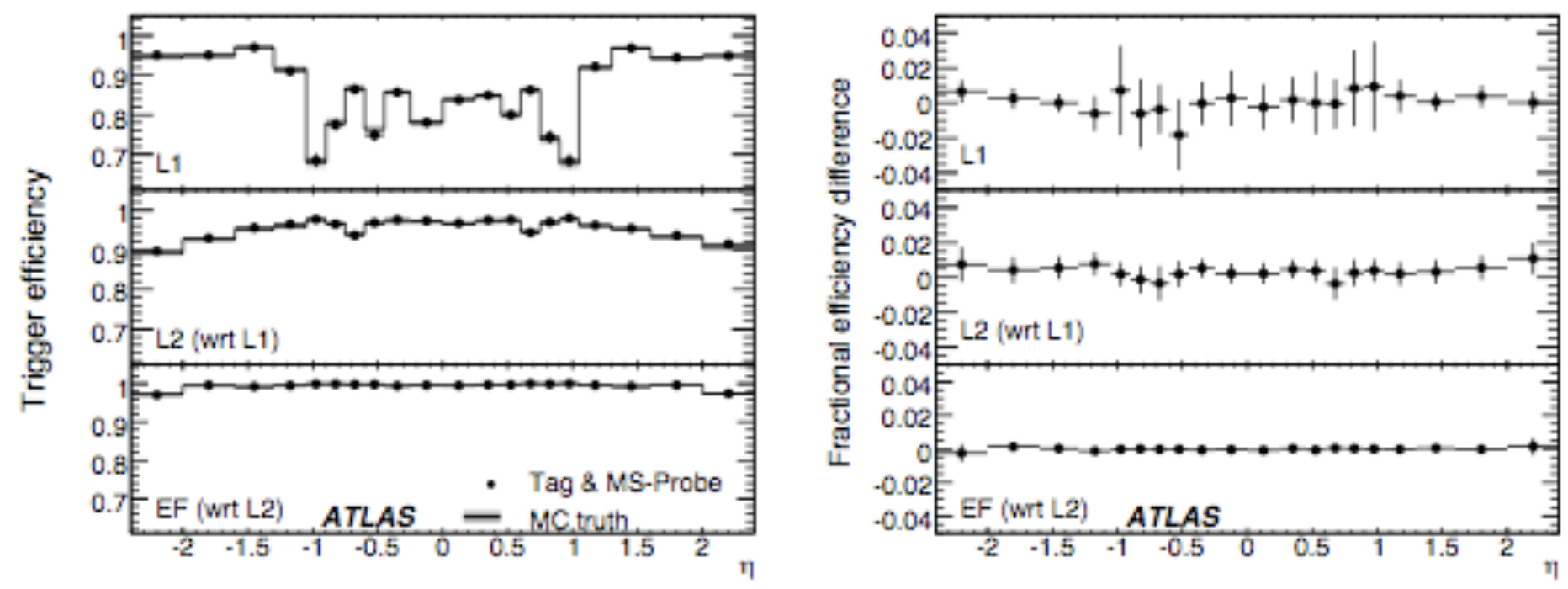


\section{e/ $\gamma$ trigger performance}

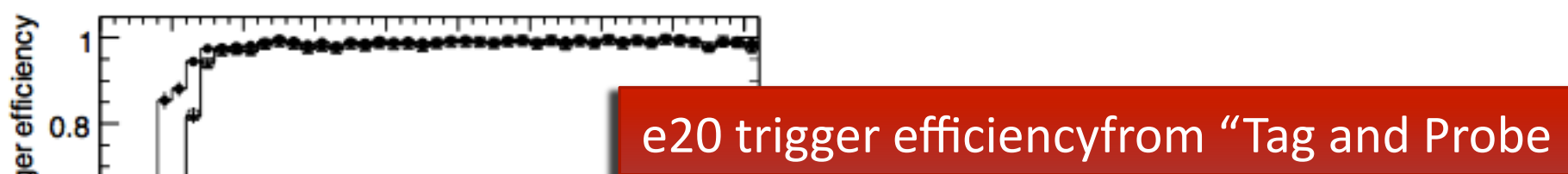

7 $e / \gamma E_{T}$ spectrum covers few GeV to several $\operatorname{TeV}(Z, J / \psi, Y$ to ee, $\gamma$-jets)

$\lambda$ Huge challenge to $L 1$ calorimeter trigger due to low $\mathrm{E}_{\mathrm{T}}$ thresholds required $(5 \mathrm{GeV}$ ) for $\mathrm{J} / \psi$ and $Y$ decay into electrons (more than $6 \mathrm{kHz}$ rate)

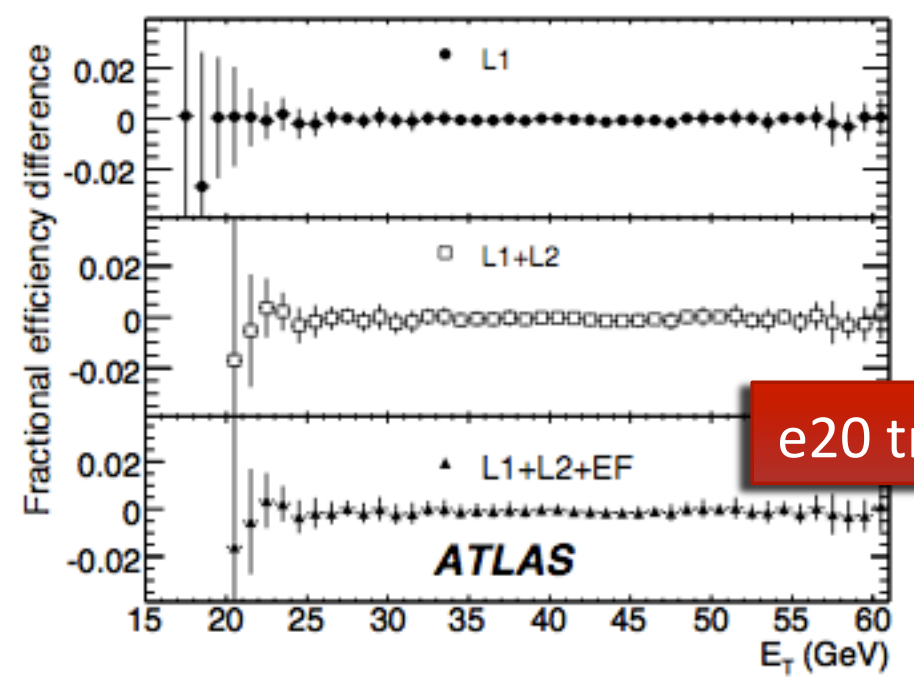




\section{ATLAS Combined performance}

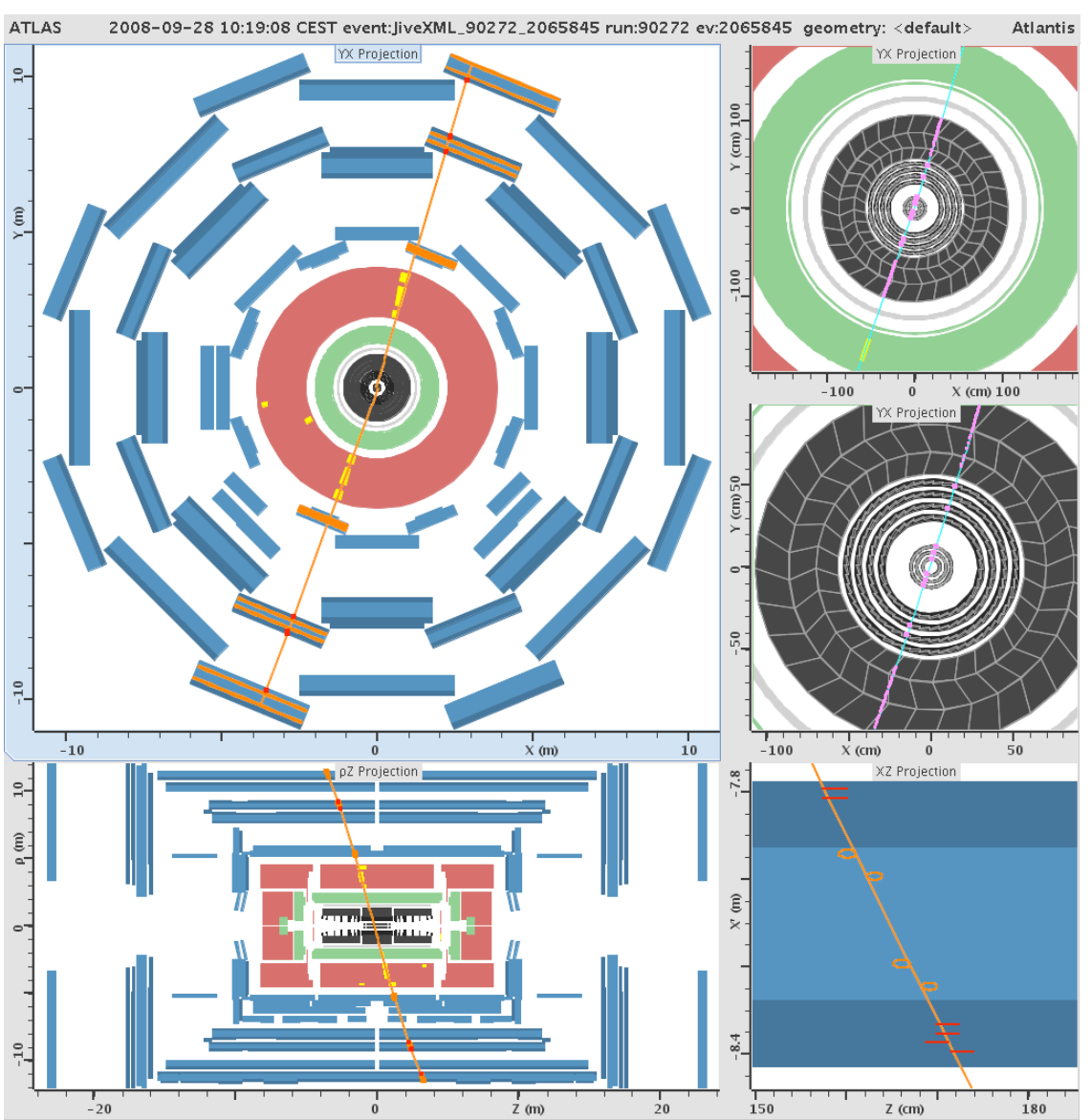

Differences in $n^{0.05}$ fíack parameters in the muon spectrometer and the ID (preliminary alignment) 等

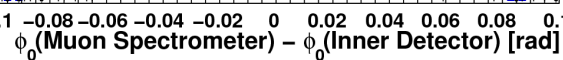

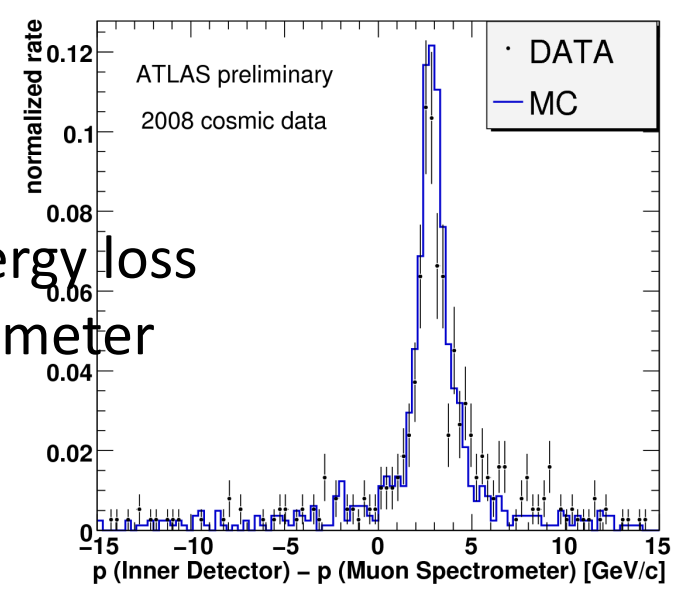




\section{Bibliography}

- http://cdsweb.cern.ch/record/1175213/files/ATL-DAQ-SLIDE-2009-106.pdf

- Multi-thread per HLT e legge di moore

- http://cdsweb.cern.ch/record/1174075/files/ATL-PHYS-SLIDE-2009-072.pdf

- Da studiare.... Rule of thumb

- http://cdsweb.cern.ch/record/1127284/files/ATL-SLIDE-2008-095.pdf

- Trigger menu, efficienza

- http://cdsweb.cern.ch/record/1095480/files/slide-2008-022.pdf

- HLt performance, trigger strategy

- http://cdsweb.cern.ch/record/1063065/files/slide-2007-054.pdf

- Trigger strategy for early running

- http://cdsweb.cern.ch/record/1049739/files/slide-2007-040.pdf

- Overall description

- http://agenda.infn.it/getFile.py/access?

contribld=114\&amp;sessionld=5\&amp;res/d=0\&amp; materialld=slides\&amp; confld=759

- Trigger strategy and menu, rates $\mathrm{L} 1$

- http://cdsweb.cern.ch/record/1133601/files/ATL-SLIDE-2008-132.pdf

- Timing I1 